\title{
Software Product Line Engineering with the UML: Deriving Products
}

Tewfik Ziadi and Jean-Marc Jézéquel

\begin{abstract}
.
Software product line engineering introduces two new dimensions into the traditional engineering of software-based systems: the variability modeling and the product derivation. The variability gathers characteristics that differ from one product to another, while the product derivation is defined as a complete process of building products from the product line. Software Product Line Engineering with the UML has received a lot of attention in recent years. However most of these works only concern variability modeling in UML static models and few works concern behavioral models. In addition, there is very little research on product derivation. This chapter investigates the product derivation in the context of the product line engineering with the UML. First, a set of extensions are proposed to model product line variability in two types of UML models: class diagrams (the static aspect) and sequence diagrams (the behavioral aspect). Then we formalize product derivation using a UML model transformation. An algorithm is given to derive a static model for a product and an algebraic approach is proposed to derive product specific statecharts from the sequence diagrams of the product line. Two simple case studies are presented, based on a Mercure product line and the banking product line, to illustrate the overall process, from the modeling of the product line to the product derivation.
\end{abstract}

\subsection{Introduction}

Rather than describing a single software system, the model of a software product line (PL) describes the set of products in the same domain. This is done by distinguishing elements shared by all the products of the line, and elements that may vary from one product to another. Concepts of commonality and variability are respectively used to designate common and variable elements in a product line [39] Variability can concern two main aspects: optionality or variation [7, 18]. An optional element only concerns some products and it can be omitted in others. Variation elements define alternatives (variants) to choose from. Beyond variability modeling, the product derivation process is defined as a complete process of constructing products from the software product line [12].

UML (Unified Modeling Language) [33] is an object-oriented notation for software system modeling. It proposes a set of models to specify several aspects of systems. Class diagrams are UML models that can be used to specify static aspects of systems, while se- 
quence diagrams and statechart diagrams are examples of models describing behavioral aspects. Software Product Line Engineering with the UML has received a lot of attention in recent years $[3,5,9,10,13,14,1826,27,37,38]$. Subchapter 999.4 presents a study on these works and shows that the most of existing works only concern UML static models and few works concern behavioral models [3, 14, 17]. In addition, there is very little research on product derivation $[3,13]$. The product derivation support is a significant criterion for determining the utility for users of any product line approach. The approaches that only model variability in UML models without product derivation support have only a descriptive utility. This means that these approaches are only useful for product line architecture description.

In this work we defend the idea that any approach of PL engineering should go beyond the descriptive utility and propose supports for resolving the variability and obtaining product models. For this, we investigate the product derivation process in the context of PL engineering with the UML. We give an overview of product line design by first presenting structural variability involved in class diagrams, then how behavioral aspects may be designed using UML sequence diagrams. We then formalize product derivation as UML model transformations. First, a transformation algorithm is given to automatically derive the static product model from the PL model. Secondly, an algebraic approach is proposed to derive product-specific statecharts from PL sequence diagrams.

To present these design techniques, subchapter 999.2 will focus on static aspects of the product line design, its constraints and its derivation process into specific products; this part also stresses the need to check derived products with respect to variability constraints. Next, subchapter 999.3 proposes an algebraic approach to derive product specific statecharts from the sequence diagrams of the product line. Here product line behaviors are specified as algebraic expressions on basic UML2.0 sequence diagrams, where variability is introduced by means of three new algebraic constructs. Our derivation approach is defined in two steps: we first define an algebraic way to derive product expressions from the PL expression and then statecharts are generated by transforming product sequence diagrams given as an expression into a composition of statecharts. Subchapter 999.4 discusses related work, and finally subchapter 999.5 draws some conclusions and perspectives.

\subsection{Deriving Static Aspects}

\subsubsection{The Mercure Product Line}

As a case study for describing static aspect derivation, we consider the Mercure PL which is a line of SMDS (Switched Multi-Megabit Data Service) servers whose design and implementation have been described in $[23,24]$. It can abstractly be described as a communication software delivering, forwarding, and relaying messages from and to a set of network interfaces connected into an heterogeneous distributed system. The Mercure PL 
must handle variants for five variation points: any number of specialized processors (Engines), network interface boards (NetDriver), levels of functionality (Manager), user interface (GUI) and support for languages (Language). Fig. 1 shows a feature diagram of the Mercure PL (we follow FODA notations [28]). The Mercure consists of Engine, Net Driver, Manager, GUI, and Language. The Mercure product may support one or more of Engine 1,..Engine N, the selection being represented by FODA alternative features. In the same way, we define all NetDriver, Manager, GUI, and Language dimensions.

The FODA [28] notations allow us to specify dependency relationships, called composition rules, between domain features. FODA supports two types of composition rules: the "require" rule that expresses the presence implication of two or more features, and the "mutually-exclusive" rule that captures the mutual exclusion constraint on feature combinations. A "require" rule is identified in the context of the Mercure PL: it specifies that the choice of the NetDriver1 implies the choice of the Engine1 (see Fig. 1).

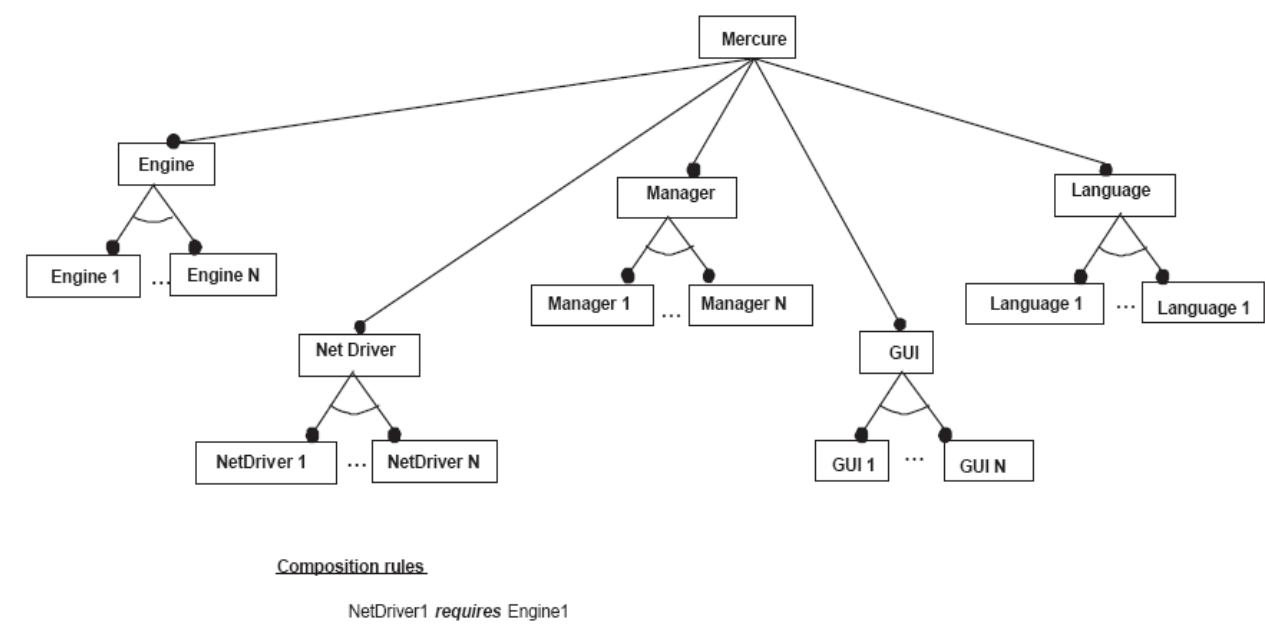

Fig. 1. The FODA diagram for the Mercure PL.

\subsubsection{PL Static architecture as UML class diagrams}

To describe the PL static architecture, we use UML class diagrams. In [42], we have proposed a UML profile for PL. This profile includes mechanisms to specify variability within two types of UML 2.0 diagrams: class diagrams and sequence diagrams. For class diagrams, we proposed to specify variability using two mechanisms: optionality and variation. 
- Optionality. Optionality in PL means that some features are optional for the PL members, i.e. they can be omitted in some products. To specify optionality in class diagrams, we introduced the <<optional>> stereotype. This stereotype can be applied to classes, packages, attributes or operations [42].

- Variation. Inheritance in UML allows defining variability in class diagrams [2]. The idea is to define a variation point as an abstract-class and variants as concrete subclasses. Each sub-class defines the implementation of the abstract-class in a specific way. However, this variability is only resolved at run-time and it is not explicit in the model. To explicitly specify the variation in UML class diagram, we introduced two stereotypes $<<$ variation $>>$ and $<<$ variant $>>$ [42]. The $<<$ variation $>>$ stereotype is associated with the abstract-class while $<<$ variant $>>$ is associated with sub-classes. Each product can choose one or more sub-classes [42]. Fig. 2 shows an example of a variation point specified using the $<<$ variation $>>$ and $<<v a r i-$ ant $>>$ stereotypes. Notice that the sub-class A in Fig. 2 is not stereotyped $<<v a r i-$ ant $>>$; this means that this sub-class is mandatory for all products.

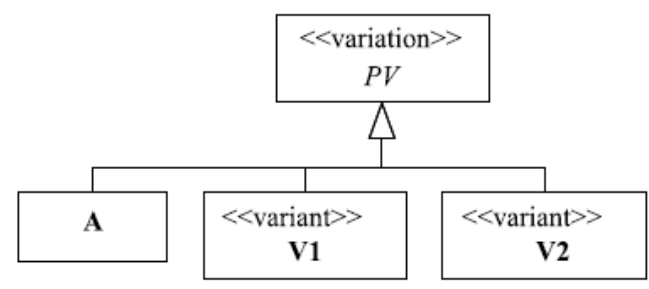

Fig. 2. Example of a variation point.

Let us now apply these extensions to the Mercure PL. As previously specified in the FODA diagram of the Mercure PL, the Mercure product may support a set of Engines among Engine1, Engine2, EngineN. Using the variation mechanism presented above, we define an abstract class called Engine and stereotyped <<variation >> and the several dimensions as sub-classes stereotyped $<<$ variant $>>$ In the same way we specify other variation points: NetDriver, Manager, GUI, and Language. Fig. 3 shows the UML class diagram of the Mercure PL. It basically says that a Mercure system is an instance of the Mercure class, aggregating an Engine (that encapsulates the work that Mercure has to do on a particular processor of the target distributed system), a collection of NetDrivers, a collection of Managers (that represent the range of functionalities available), and the GUI that encapsulates the user preference variability factor. A GUI has itself a collection of supported languages (see Fig. 3). 


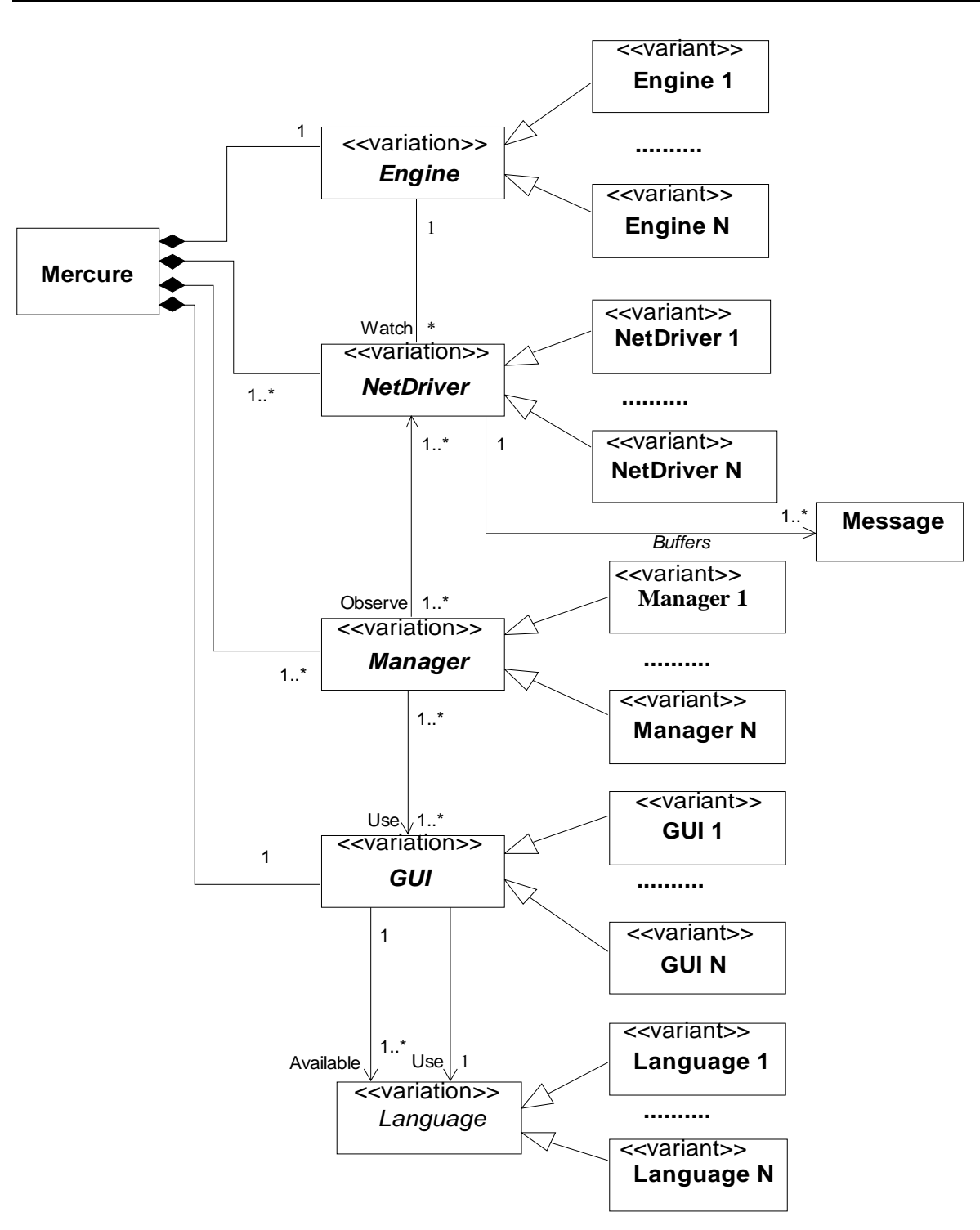

Fig. 3. The Mercure Product Line UML class diagram.

\subsubsection{Product Line Constraints}

In addition to variability, the product line architecture is defined as a standard architecture with a set of constraints [4]. In this context, we have identified in [45] two types of PL constraints that guide the product derivation process. We proposed to define them as OCL 
(Object Constraints Language) meta-level constraints. In what follows we briefly present both the generic constraints that apply to all product lines, and specific constraints that concern a specific PL (a detailed description of these constraints can be found in [45]).

\subsubsection{Generic Constraints}

The introduction of variability using the $<<$ variant $>>$, $<<$ variation $>>$, and $<<$ optional >> stereotypes improves genericity but can generate some inconsistencies. For example, if a mandatory element depends on an optional or on a variant one, the derivation can produce an incomplete product model. So the derivation process should preserve the consistency of the derived products. In [45], we proposed the formalization of consistency constraints using OCL and we called them Generic Constraints. An example of such constraint is the dependency constraint that forces mandatory elements to depend on mandatory ones only. It is specified using OCL as the following invariant for the Dependency ${ }^{1}$ metaclass:

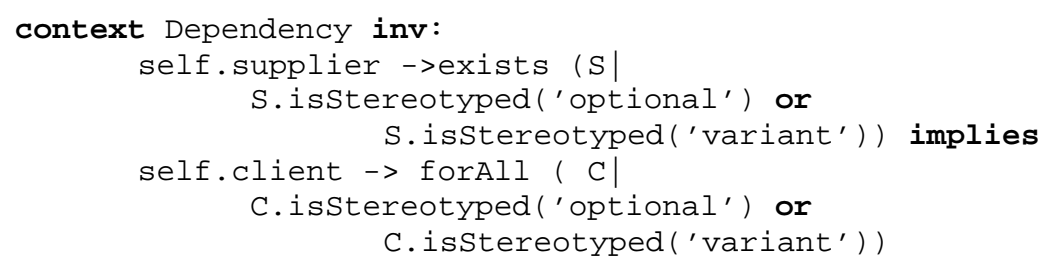

isstereotyped(S) is an auxiliary primitive indicating if an element is stereotyped by a string S. It is formalized using OCL as follows:

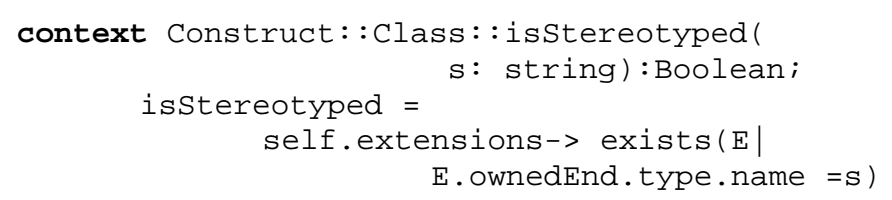

\subsubsection{Specific constraints}

A fundamental characteristic of the product line is that all elements are not compatible. That is, the selection of one element may disable (or enable) the selection of others. For example in the class diagrams for the Mercure PL in Fig. 3, the choice of the class variant NetDriver1 in the specific product needs the presence of the Engine1 variant. Another challenge for the product derivation is to ensure these dependencies in the derived products. In our work, these dependencies are called Specific Constraints and are also

\footnotetext{
${ }^{1}$ A dependency in the UML specifies a require relationship between two or more elements. It is represented in the UML meta-model [33] by the meta-class Dependency; it represents the relationship between a set of suppliers and clients. An example of the UML Dependency is the "Usage", which appears when a package uses another one.
} 
formalized as OCL meta-level constrains [45]. The presence constraint in the Mercure PL is formalized as an invariant for the Model meta-class as follows:

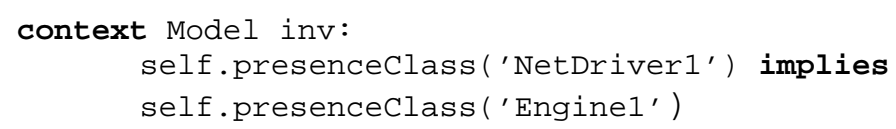

presenceclass(C) is an auxiliary operation indicating if a specific class called $\mathrm{C}$ is present in the model. It is formalized using OCL as follows:

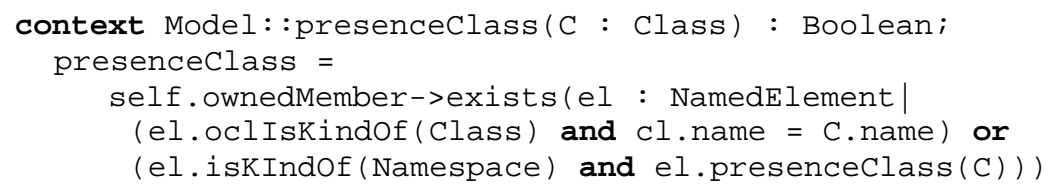

\subsubsection{From Product Line models to Product Models}

Deriving static aspects in PL consists in generating the UML class diagram of each product from the PL class diagram. As shown previously, the PL class diagram is defined by a set of variation points and to derive a specific-product class diagram, some decisions (or choices) associated with these variation points are needed. For example, each Mercure product could choose among the presence or absence of all variant classes. A mechanism is needed to capture the decisions that are made for a specific product. As in [3], we call this mechanism a decision model. In this subchapter, we propose to use the Abstract Factory design pattern as a decision model associated with the PL class diagram. Then we propose an algorithm, based on models transformation, to derive product class diagrams. To illustrate this algorithm, we use three products in the Mercure PL: FullMercure, CustomMercure and MiniMercure while,

- FullMercure is the product that includes all NetDrivers; all Engines; all Managers; all GUIs; all Languages. Thus, all combinations can be dynamically bound.

- CustomMercure is a restricted product. It only supports two different network drivers : NetDriver1 and NetDriver2; one manager: Manager1; two GUIs: GUI1 and GUI2; two languages: Language1, Language2;

- MiniMercure] is a lightest product that only supports NetDriver1, Engine1, GUI1, Manager1, and Language1.

\subsubsection{The Decision Model}

The Abstract Factory is a creational design pattern [15]. It allows defining an interface for creating a line of related objects. In [25], one of the authors proposed the use of this pattern to refine product derivation at compilation time. Our aim in this subsection is to reuse again this pattern as a design of the PL decision model. Fig. 4 shows the structure of our decision model applied to the Mercure PL. We use an abstract factory, called Mer - 
cure_Factory to define an interface for creating variants of Mercure's five variation points. The abstract class Mercure_Factory defines five factory methods, one for each variation point. new_gui ( ) for example is the factory method which concerns the GUI variation point. These factory methods are abstractly defined in the class Mer cure_Factory, and given concrete implementation in its sub-classes called concrete factories. We create one concrete factory for each product in the PL. FullMercure, CustomMercure and MiniMercure in Fig. 4 are concrete factories for the Mercure PL. We propose to specify decisions related to each product using stereotypes applied to method factories. We use stereotypes to restrict the return type of factory methods to the possible one. For example, the CustomMercure product model includes only GUI1, and GUI2. The Factory Method that corresponds to the GUI variation point is new_gui( ), so we add two stereotypes $<<$ GUI1 $>>$ and $<<$ GUI2 $>>$ to this factory method (see Fig. 4 ).

\subsubsection{Derivation}

Now we have to tackle the automation of the derivation process exploiting the variation points and the decision model. The derivation algorithm we use to derive product models is described in Fig.5. It takes as input the PL class diagram, and the concrete factory from the decision model and it generates as output the product class diagram. It is decomposed into three steps: selection of variant classes, model specialization, and model optimization. The next paragraphs briefly describe each step.

- $\quad$ Step 1: Variant classes selection. The first step consists of selecting variant classes using the concrete factory. For each factory method, we retrieve its stereotypes. These stereotypes define the names of the selected sub-classes of the abstract-class returned by the factory method. When the factory method does not define stereotypes (such as in the FullMercure concrete factory methods), all the sub-classes of its return type are selected.

- $\quad$ Step 2: Model specialization. In this step, we remove all variants classes from the model which have not been selected in the first step. However, to preserve coherence, variant ancestors of selected variant elements are not removed.

- Step 3: Model optimization. Here we delete unused factories and optimize the inheritance. Inheritance optimization is applied when there is only one concrete class inheriting from an abstract one. In this case the abstract-class is omitted and replaced by the concrete one. 


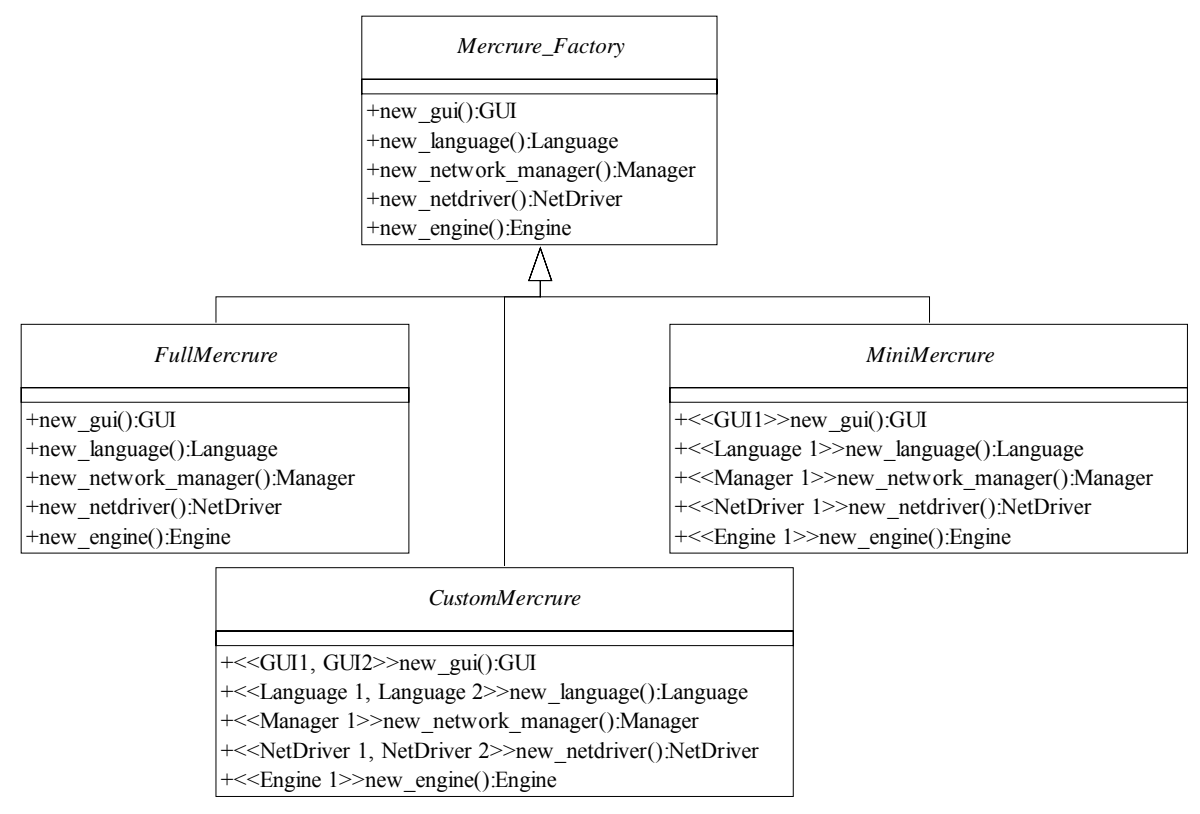

Fig. 4. The Abstract Factory as a decision model for the Mercure PL. 


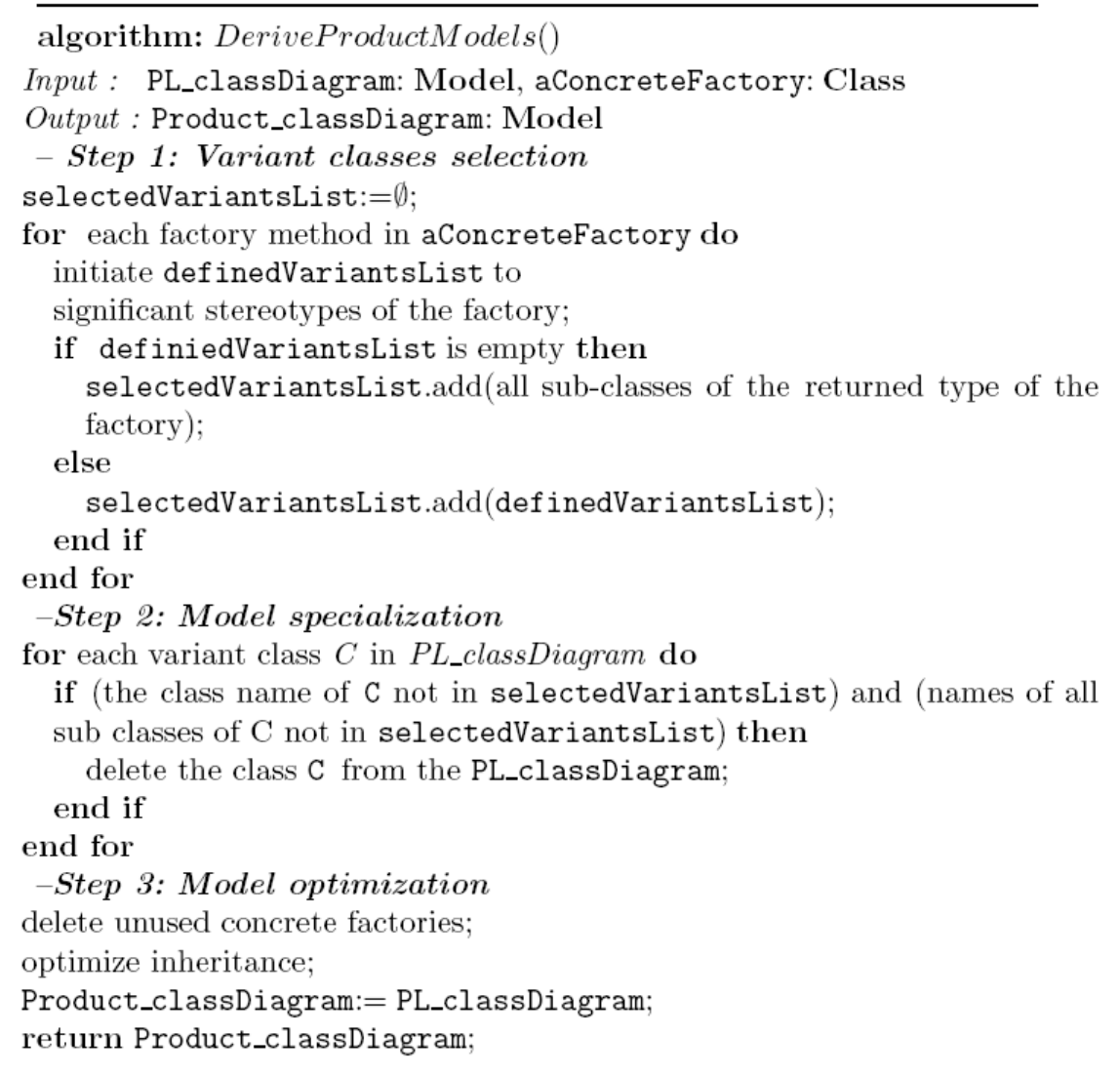

Fig. 5. Static aspect derivation: the derivation algorithm.

To achieve the implementation of the derivation algorithm, we have used the INRIA Model Transformation Language (MTL). Information about implementation and technical materials can be found at http://modelware.inria.fr/mtl. We have applied the derivation for the three Mercure products: FullMercure, CustomMercure, and MiniMercure. Fig. 6 shows the CustomMercure model obtained by derivation from the Mercure model in the Fig. 3. 


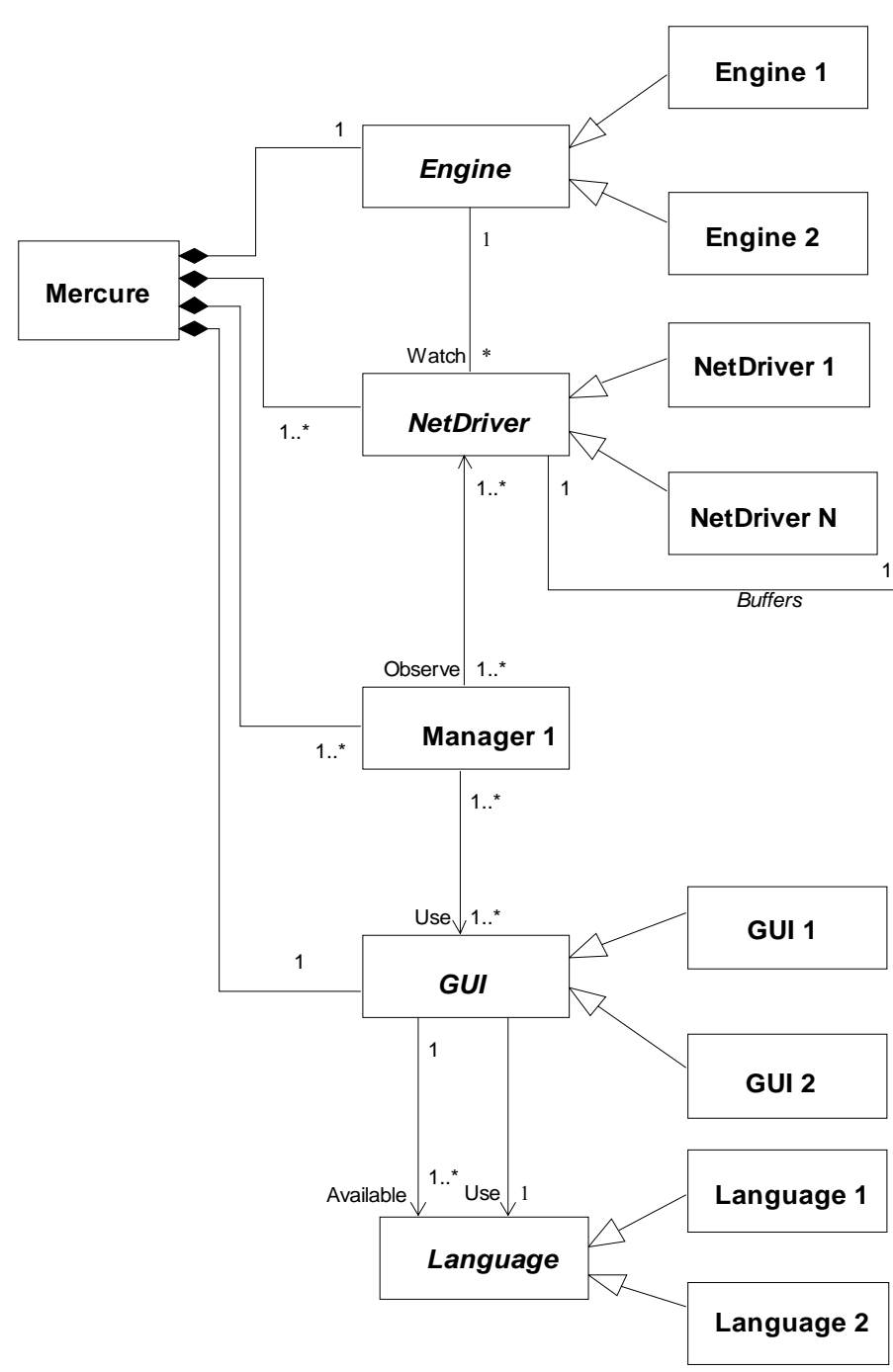

Fig. 6. The CustomMercure model, automatically derived from the Mercure PL model.

\subsubsection{Derivation versus constraints}

The product line model should satisfy generic constraints before the derivation and the product model derived should satisfy specific constraints. The generic constraints represent the pre-conditions of the derivation algorithm while specific constraints represent the post-conditions: 


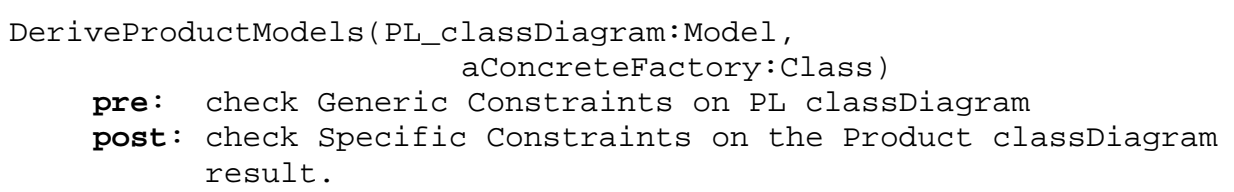

\subsection{Deriving Behavioral Aspects}

In addition to static aspect description, behavior modeling plays an important role in the traditional engineering of software-based systems; it is the basis for systematic approaches to requirements capture, specification, design and simulation, code generation, testing, and verification. Scenario languages such as UML2.0 Sequence Diagrams (SD) are an example of formalisms for modeling behavior. They focus on the global interactions between actors and system components. To be useful in the PL context, sequence diagrams should also allow for expression of variability. We show in this subchapter how variability can be expressed in UML2.0 sequence diagrams using UML stereotypes and tagged values. We take advantage of UML2.0 SDs and their composition operators to specify PL sequence diagrams as algebraic expressions extended by algebraic constructs for variability. Then we present an algebraic approach to derive the product behaviors from the PL sequence diagrams. Before illustrating behavioral aspect derivation, we briefly present the Banking Product Line (BPL) as an example which will be used throughout this subchapter.

\subsubsection{The Banking Product Line}

In this subchapter, we reuse the example of a Banking Product Line (BPL) as described in [3]. It is a set of products providing simple functionalities to clerks in the banking domain. It provides four main functionalities:

- Creation of accounts (F1) Customers are able to open simple accounts but must do so with a minimum balance. Account can have an associated limit specifying to what extent a customer can overdraw money.

- Money deposit on accounts (F2) Customers can deposit an amount of money on their accounts.

- Money withdrawal from accounts (F3) Customers can withdraw money from their account. If the account has a limit, a customer can only withdraw money up to this limit. If not, he(or she) cannot withdraw beyond the current balance of the account.

- Currency exchange calculation (F4) The bank system can offer a functionality for exchange calculation. This particulary concerns currency exchange: euros, dollars, etc.

Variability in the BPL example concerns the support of overdrawing to a set limit which is optional because some products do not allow the addition of limits on accounts. Currency exchange calculation is also an optional functionality and it is only supported by some products. Table. 1 shows four different product members of the BPL. The BS1 product for example supports limits on accounts and does not support exchange calcula- 
tion while BS4 is a complete product with limits on accounts and exchange calculation support.

Table 1. The Banking PL Members.

\begin{tabular}{|l|l|l|}
\hline Product & Limit support & Exchange calculation \\
\hline BS1 & YES & NO \\
BS2 & NO & NO \\
BS3 & NO & YES \\
BS4 & YES & YES \\
\hline
\end{tabular}

\subsubsection{Product Line Behaviors as UML2.0 Sequence Diagrams}

\subsubsection{UML2.0 sequence diagrams}

UML2.0 sequence diagrams [33] enhance the previous versions of scenarios proposed in UML1.x by introducing composition operators. A basic sequence diagram describes a finite number of interactions between a set of objects. The semantics of a basic SD is now based on partially ordered events (instead of ordered collections of messages as in UML1.x), which makes it easy to introduce concurrency and asynchronism, and allows the definition of more complex behaviors.

Fig. 7 shows the basic SD related to the Banking PL. A UML2.0 SD is represented by a rectangular frame labeled by the keyword sd followed by the name of the SD. The SD Deposit for example shows interactions between Clerk, Bank and Account to deposit an amount on a specific account. The vertical lines represent life-lines for the given objects. Interactions between objects are shown as horizontal arrows called messages (like deposit). Each message is defined by two events: message emission and message reception, which induce an ordering between emission and reception. Events located on the same lifeline are ordered from top to down. 


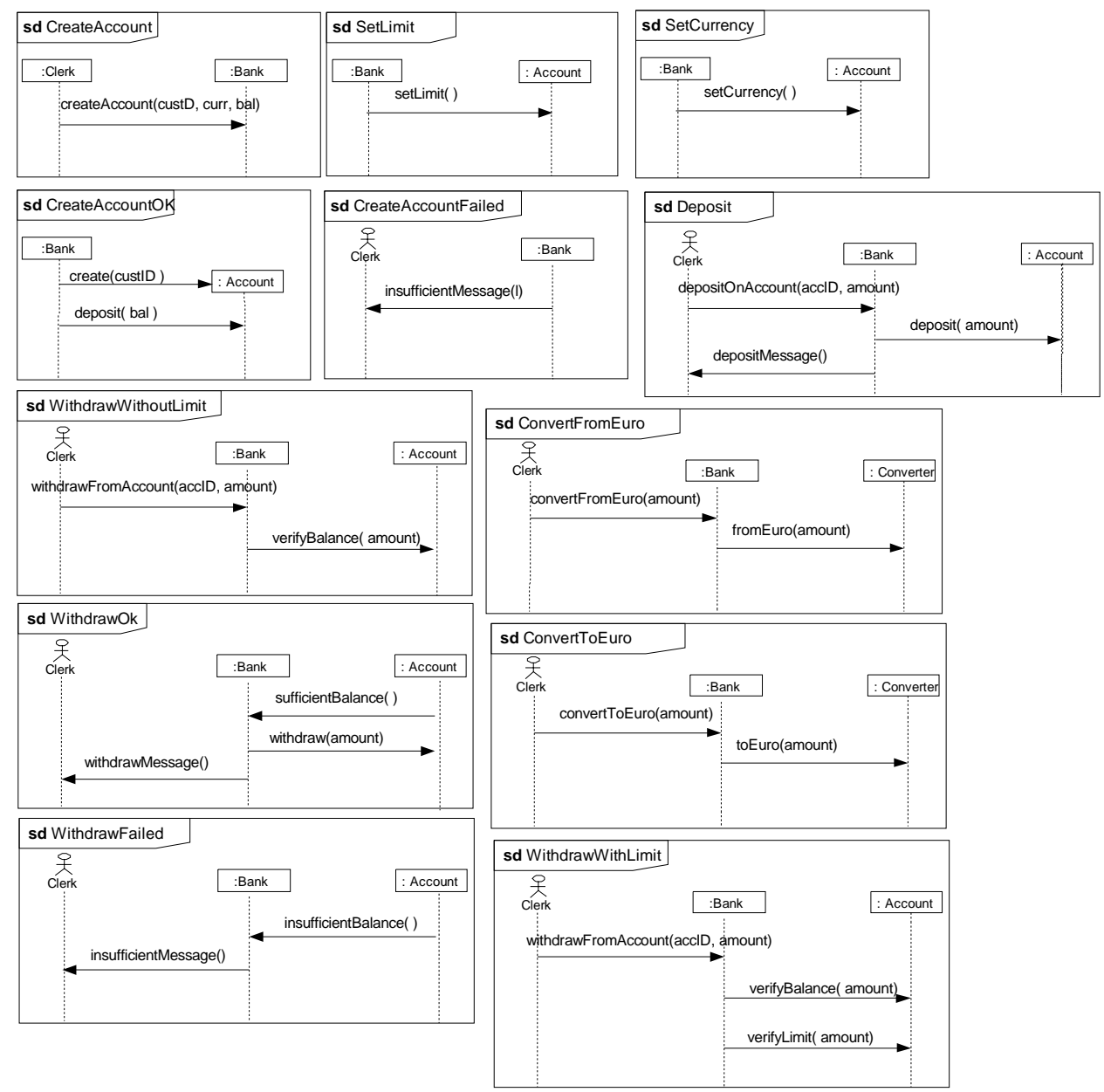

Fig. 7. UML2.0 Sequence Diagrams for the Banking PL.

UML2.0 basic SD can be composed into composite SDs called combined interactions using a set of operators called interaction operators [33]. We will only use three fundamental operators: seq, alt, and loop. The seq operator specifies a weak sequence ${ }^{2}$ between the behaviors of two operand SDs. The alt operator defines a choice between a set of interaction operands. The loop operator specifies an iteration of the SD. For all these operators, each operand is either a basic or a combined SD. The combined SD BankPL in Fig. 8 shows how basic SDs for the BPL are related. It refers to the basic interactions us-

${ }^{2}$ UML2.0 [33] defines two operators, seq and strict to define respectively weak and strict sequence. A weak sequence means that only events on the same lifeline in the first SD are executed before events on the same lifeline in the second SD. A strict sequencing means that all events in the first SD are executed before events in the second diagram. 
ing the ref operator. BankPL specifies that there are five main alternative behaviors for requirements of BPL members: (1) Account creation. (2) Deposit on account. (3) Withdraw from account (this last functionality is described using the combined SD WithdrawFromAccount). (4) Exchange calculation from Euro. (5) Exchange calculation to Euro. Following UML2.0 notations [33], combined SDs are defined by rectangles whose left corner is labelled by an operator (alt, seq, loop). Operands for sequence and alternative are separated by dashed horizontal lines. Sequential composition can also be implicitly given by the relative order of two frames in a diagram. For example, in the SD BankPL basic SD CreateAccountok is referenced before SD SetLimit. This is equivalent to the expression CreateAccountok seq SetLimit.

\subsubsection{Variability in Sequence Diagrams}

As shown in [42, 43], variability can be specified in UML2.0 sequence diagrams using simple stereotypes and tagged values. We briefly describe here these mechanisms, interested readers can refer to $[42,43]$ for more detail:

- Optionality. A combined SD can refer to an optional SD: interactions specified by this optional SD are only supported by some products and can be omitted in others. To specify optionality of a SD, we introduced the <<optionalinteraction>> stereotype and the optionalPart tagged value. The tagged value specifies the occurrence name of the optional SD (to differentiate among various occurrences of the optional SD, since an optional SD might be referred more than once in the same combined SD). Fig. 8-a shows an example of a combined SD called CDS1 which refers to an optional SD called SD1. The tagged value optionalPart takes SD1-occ1 as value $^{3}$.

- Variation. This variability mechanism makes it possible to define a set of variants of behaviors from which a particular product would have to select exactly one variant. Using UML2.0 SDs, the variation of the behavior is modeled as a combined SD stereotyped $<<$ variation $>>$ which refers to a set of sub-interactions stereotyped $<<$ va riant $>>$. Each sub-interaction specifies a variant behavior. As for the optional SD, a variation $\mathrm{SD}<<$ variation $>>$ can be referred to several times in the same combined SD. To differentiate among multiple occurrences, we introduce the tagged value variationPart to specify the name of the occurrence. Fig. 8-b shows an example of a variation SD called CSD2 which refer to two SD variants SD-V1 and SD-V1. Note that this variation mechanism is different from the alt interaction operator. The variation mechanism proposes a choice that must be made at product derivation time so that the derived product contains only one of the alternative behaviors, while the alt operator defines a choice made after the product derivation, i.e. at run-time.

\footnotetext{
${ }^{3}$ We follow new notations of tagged values in UML2.0: a tagged value is now represented in UML2.0 as a note [33].
} 
- Virtuality. The virtuality of a SD means that its behavior can be redefined by another $\mathrm{SD}$ or refinement associated to a specific product. This type of variability is inspired by an existing construction in MSC [22]. The behavior of the virtual SD will be replaced at product derivation time by the behavior of the refinement SD associated with the product. Virtuality is introduced by the stereotype <<virtual >> and the tagged value virtualpart indicating the occurrence of the virtual interaction. Fig. 8-c shows an example of a combined SD called CSD3 which refers to a virtual SD called SD3.

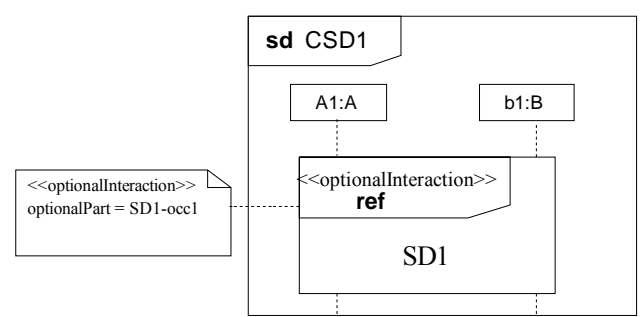

(a) Optionality

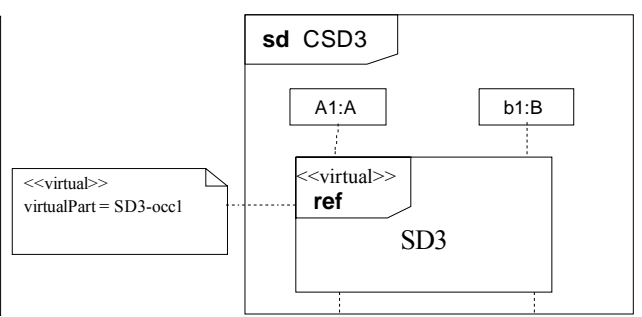

(c) Virtuality

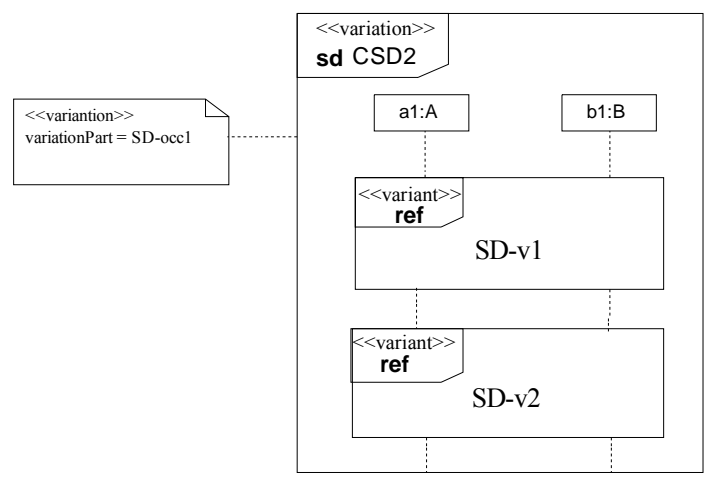

(b) Variation

Fig. 8. Variability for UML2.0 SD.

The combined SD in Fig. 9 BankPL illustrates two variability mechanisms: optionality and variation.

1. Since some products of the BPL do not support overdrawing, a stereotype $<<$ op tionalInteraction>> is added to the basic SD SetLimit and the tagged value optionalPart takes the value settingLimit (see the combined SD AccountCreation in Fig. 9). In addition, since exchange calculation is an optional functionality in the BPL, basic SD SetCurrency, ConvertToEuro and ConvertFromEuro are defined as optional too (see the combined SD AccountCreation in Fig. 9. 
2. There are two SD variants when withdrawing from an account: withdraw with balance and limit checking, and withdraw with balance checking only. The SD Withdraw is defined with the <<variation $>>$ stereotype. The two SDs WithdrawWithLi mit and WithdrawWithoutLimit are stereotyped $<<$ variant $>>$. The tagged value variationPart takes withdrawAccount as value (see the WithdrawFromAccount combined SD in the Fig. 9).

\subsubsection{Algebraic Specification}

Taking advantage of UML2.0 composition operators for SD, we introduce in this subchapter an algebraic specification of UML2.0 SDs in the form of reference expressions. We then extend it for product lines by including variability constructions defined above.

Definition 1. A reference expression for sequence diagrams (noted RESD hereafter) is an expression of the form:

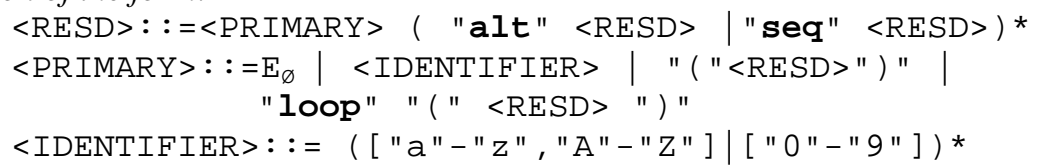

seq, alt and loop are the $S D$ operators mentioned above. $\mathrm{E}_{\emptyset}$ is the empty expression that defines a sequence diagram without interaction.

So far, this algebraic framework does not contain any means to specify variability. We introduce three algebraic constructs that correspond to the three variability mechanisms presented above. This allows the definition of optional, variation and virtual expressions. 
18 Tewfik Ziadi, Jean-Marc Jézéquel
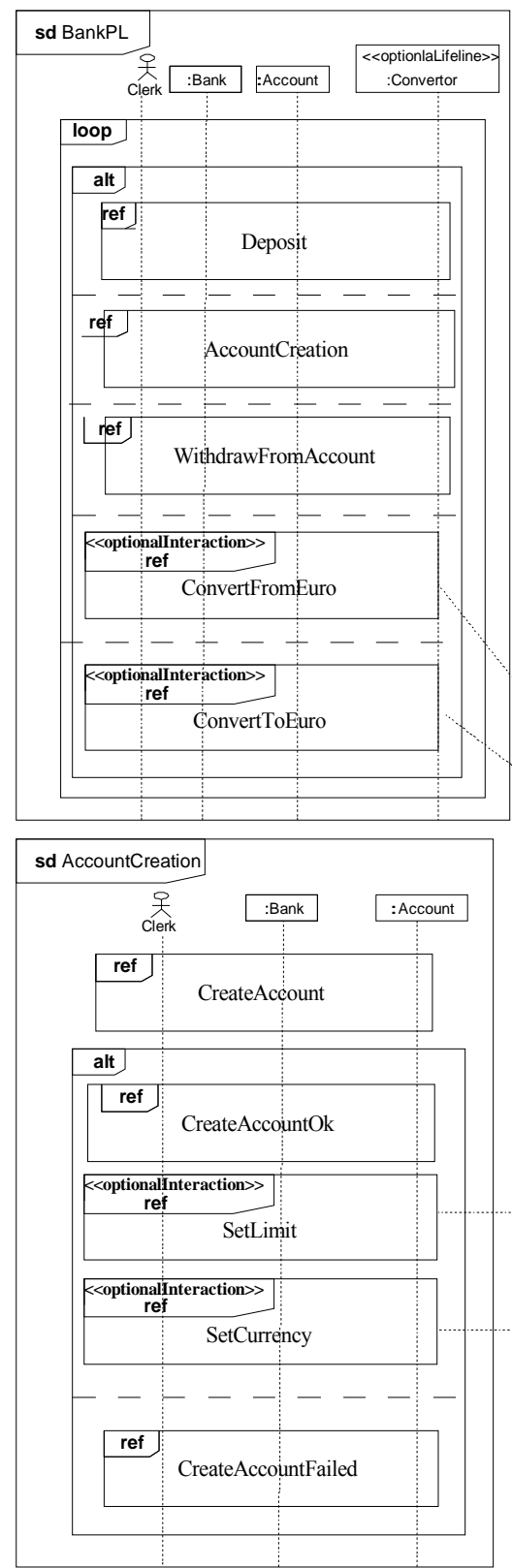
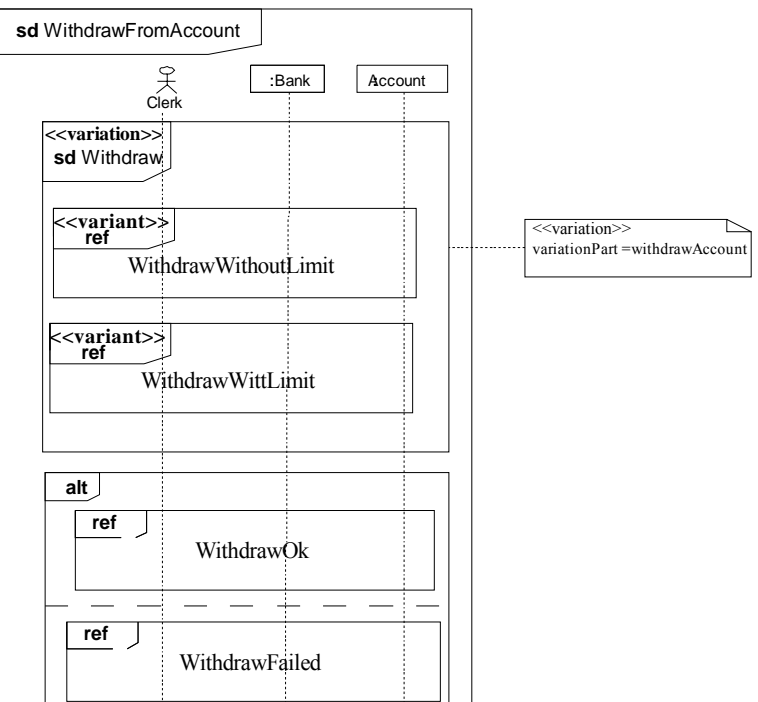

$<<$ optionalInteraction $>>$ optionalPart = fromEuro

$<<$ optionalInteraction $>>$ optionalPart =toEuro <<optionallnteraction $>>$ optionalPart =settingLimit

<<optionalInteraction $>>$ optionalP art =settingCurrenc

Fig. 9. The UML2.0 Combined Sequence Diagram for the Banking PL. 
Definition 2. The optional expression $(O p E)$ is specified in the following form:

$$
\text { OpE : := "optional" <IDENTIFIER> "[" <RESD> "]" }
$$

where $<$ IDENTIFIER $>$ refers to the name of the optional part and the $<\mathrm{RESD}>$ refers to its corresponding expression.

An optional SD (i.e. a SD stereotyped <<optionalInteraction>>) can be specified by an optional expression. The tagged value optionalpart in the diagram specifies the name of the expression. For the BPL example, optionality of the interaction SetLimit is specified by the expression:

\section{optional settingLimit [ SetLimit]}

Definition 3. A Variation expression (VaE) is defined as follows:

VaE: :="variation" <IDENTIFIER> "[" <RESD> "," ( <RESD>)* "]"

For example, the variation interaction Withdraw in Fig. 9 encloses two interaction variants. It is specified algebraically as follows:

variation withdrawAccount [ WithdrawWithLimit, WithdrawWithoutLimit ]

Definition 4. Virtual expressions (ViE) are specified as:

ViE : := "virtual" <IDENTIfIER> "[" <RESD> "]"

Hence, algebraic expressions including variability will be defined by expressions of the form:

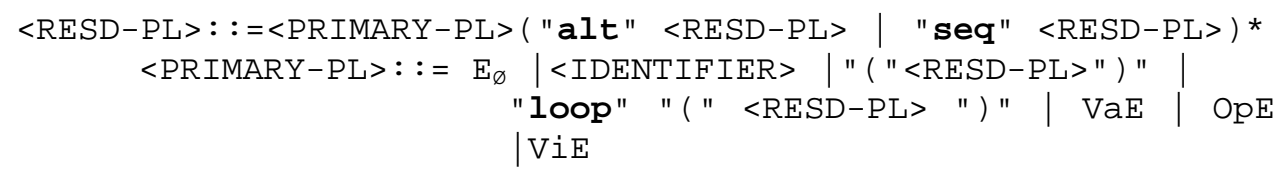

The SD BankPL of Fig. 9 can be algebraically represented by the following expression:

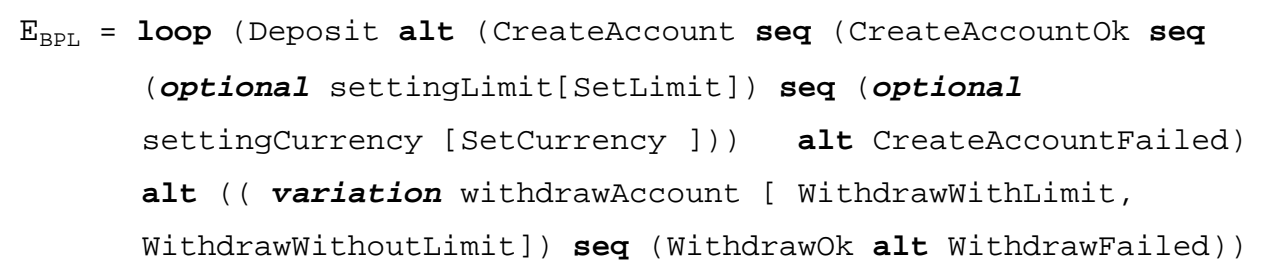


alt (optional fromEuro [ ConvertFromEuro ])

alt (optional toEuro [ ConvertToEuro ] ))

\subsubsection{Deriving Product Behaviors}

In the previous subsection, we have specified PL behaviors using scenarios represented as UML2.0 SD enriched with variability mechanisms. Scenarios are not the only way to describe software behaviors; statecharts [19] are another formalism that is often used to depict the behavioral aspects of systems. However, if scenarios capture requirements in the early stage of the development process, statechart models are more dedicated to detailed design phases as they are closer to the implementation (some tools such as Rhapsody [21] generate code from them). To formalize product behavior derivation, we have studied the problematic of statechart synthesis from scenarios. Furthermore, scenarios and statecharts differ in their nature (scenarios capture interactions amongst a set of objects, and statecharts represent the internal behavior of a single object). Statechart synthesis out of a collection of scenarios has received a lot of attention in the context of single product development $[29,30,32,40]$. So far, the proposed solutions do not consider the PL aspects. In this subchapter, we propose an algebraic approach to synthesize product statecharts from PL scenarios. Firstly, variability is resolved by deriving the RESD-PL into a set of RESDs, one for each product. Then statecharts are generated by transforming product scenarios given as an RESD into a composition of statecharts.

\subsubsection{Step 1: Product expressions derivation}

The first step towards product behavior derivation is to derive the corresponding product expressions from the RESD-PL. Decision resolutions for a specific product are defined in what we call an Instance of Decision Model, which is defined as follow:

Definition 5. An Instance of Decision Model (noted hereafter IDM) for a product $\mathrm{P}$ is a set of pairs ( name $_{i}$, Res), name ${ }_{i}$ designates a name of an optional, variation or virtual part in the RESD-PL and Res is its decision resolution related to the product $\mathrm{P}$. Decision resolutions are defined as follows:

- The resolution of an optional part is either TRUE or FALSE.

o For a variation part with $E_{1}, E_{2}, E_{3}$.. as expression variants, the resolution is $i$ if $E_{i}$ is the selected expression.

o The resolution of a virtual part is a refinement expression $E$.

Table. 2 shows four Instances of Decision Model associated with the four products in the Banking Product Line. For example, IDM1 is the Instance of Decision Model associated with the product BS1 which supports limits on accounts and does not offer the currency exchange calculation functionality.

The derivation can be seen as a model specialization through abstract interpretation of a generic PL expression in the IDMi context, where IDMi is the Instance of Decision 
Model related to a specific product. For each variability mechanism, the interpretation in a specific context is quite straightforward:

- Interpreting an optional expression means deciding on its presence or absence in the product expression. This is defined as:

$[[\text { optional name }[\mathrm{E}]]]_{\mathrm{IDMi}}=\left\{\begin{array}{l}\text { E if }(\text { name, TRUE }) \in \text { IDMi } \\ \text { E }_{\emptyset} \text { if }(\text { name, FALSE }) \in \text { IDMi }\end{array}\right.$

Note that the empty expression is a neutral element for the sequential and the alternative composition. It is also idempotent for the loop, i.e:

$$
\begin{array}{ll}
\text { - } & E \text { seq } E_{\emptyset}=E ; E_{\varnothing} \text { seq } E=E \\
\text { - } & E \text { alt } E_{\varnothing}=E ; E_{\varnothing} \text { alt } E=E \\
\text { - } \operatorname{loop~}\left(E_{\varnothing}\right)=E_{\varnothing}
\end{array}
$$

This allows us to replace a complete part of a RESD-PL by $E_{\varnothing}$ when this part should be removed.

- Interpreting a variation expression means choosing one expression variant among its possible variants. This is defined as:

$\llbracket[\operatorname{variation} \text { name }[E 1, E 2, \ldots]]_{I D M i}=E j$ if $($ name,$j) \in$ IDMi

- Interpreting virtual expressions means replacing the virtual expression by another expression:

\begin{tabular}{|c|c|}
\hline Product & Instance of Decision Model (IDM) \\
\hline BS1 & $\begin{array}{l}\text { IDM1 }=\{(\text { settingLimit, TRUE), ( settingCurrency, } \\
\text { FALSE), (withdrawAccount, } 1),(\text { fromEuro, FALSE), } \\
(\text { toEuro, FALSE) }\}\end{array}$ \\
\hline BS2 & $\begin{array}{l}\text { IDM2 }=\{(\text { settingLimit, FALSE), ( settingCurrency, } \\
\text { FALSE),(withdrawAccount, } 2),(\text { fromEuro, FALSE), } \\
(\text { toEuro, FALSE) }\}\end{array}$ \\
\hline BS3 & $\begin{array}{l}\text { IDM3 }=\{(\text { settingLimit, FALSE), } \\
\text { FALSE), (withdrawAccount, 2), (fromEuro, TRUE), } \\
(\text { toEuro, TRUE) }\}\end{array}$ \\
\hline
\end{tabular}

$$
[\text { virtual name }[E]] \text { IDMi }=E^{\prime} \text { if }\left(\text { name, } E^{\prime}\right) \in \operatorname{IDMi}
$$

Table 2. Instances of the decision model for the banking product line. 


\begin{tabular}{|c|c|c|}
\hline BS4 & $\begin{array}{l}\text { IDM4 }=\{\text { ( settinglimit, } \\
\text { TRUE), (withdrawAccount, } \\
\text { (toEuro, TRUE) }\}\end{array}$ & $\begin{array}{l}\text { TRUE), ( settingCurrency, } \\
1), \text { (fromEuro, TRUE), }\end{array}$ \\
\hline
\end{tabular}

The $B S 2$ product expression $E_{B S 2}$ is obtained by the interpretation of the $E_{B P L}$ in the IDM2 context:

$$
\mathrm{E}_{\mathrm{BS} 2}=\left[\left[\mathrm{E}_{\mathrm{BPL}}\right]\right]_{\text {IDM2 }}
$$

The derivation of the four optional expressions and the variation expression in $\mathrm{E}_{\mathrm{BPL}}$ is realized as follows :

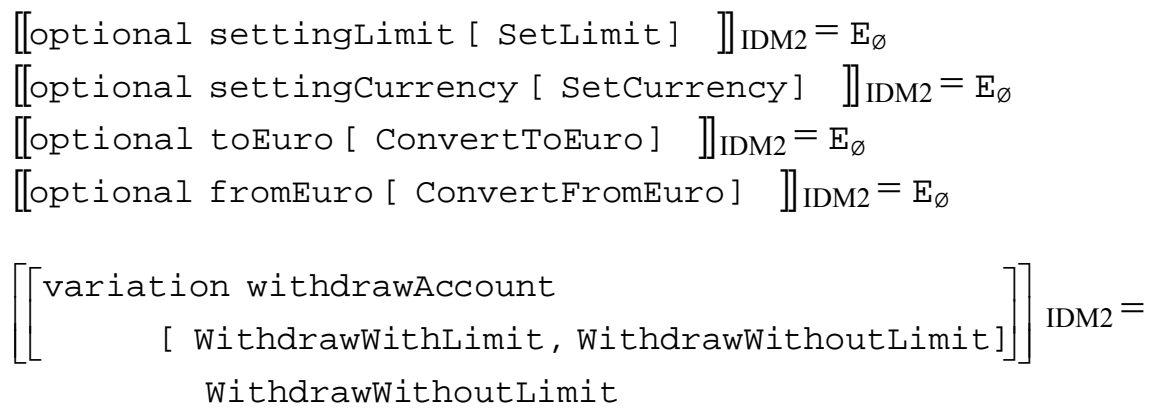

The reference expression obtained for the BS2 is the expression $E_{B S 2}$ below. Since $E_{\emptyset}$ is a neutral element for seq and $\mathbf{a l t}, \mathrm{E}_{\emptyset}$ is removed from the product expression:

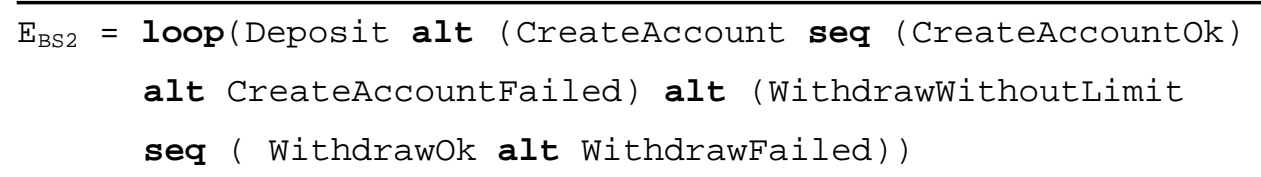

The BS4 product, which provides overdrawing on accounts and exchange operations, will be characterized by the presence of SetLimit, SetCurrency, Convert ToEuro, and Conver tFromEuro SDs; and by the choice of WithdrawWithLimit $\mathrm{SD}$. The product expression obtained for product BS4 is:

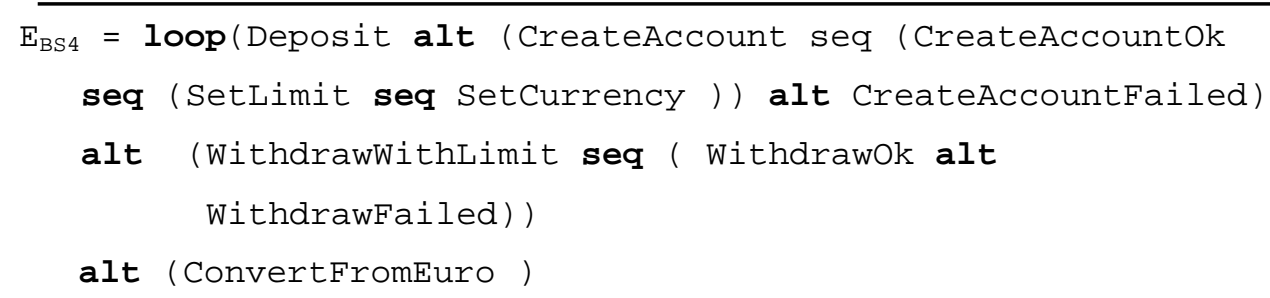


alt (ConvertToEuro)

\subsubsection{Step 2: Statechart Synthesis}

The derived product expressions are expressions without variability, i.e. expressions that only compose basic SDs by interaction operators: alt, seq, and loop. The second step of our derivation approach aims at generating statecharts for objects in each derived product. Product sequence diagrams are translated into statecharts using the method proposed in [44]. We generate flat statecharts, i.e. statecharts without hierarchy. Fig. 10 shows examples of flat statecharts, in which states represented by double circled states are called junction states. Junction states are introduced to formalize statechart composition [44]. Transitions are labeled $\mathrm{e} / \mathrm{a}$ where $\mathrm{e}$ is a triggering event and $\mathrm{a}$ is an action. $\mathrm{ST}_{\varnothing}$ refers to an empty statechart, containing a single state which is at the same time an initial and a junction state (see the $\mathrm{ST}_{\varnothing}$ statechart in Fig. 10).

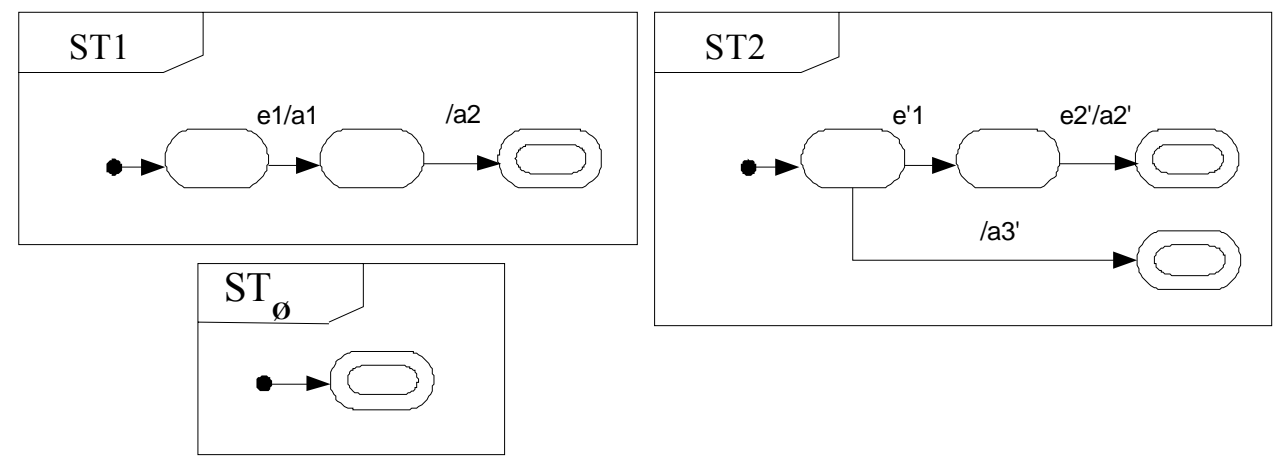

Fig.10. Example of flat statecharts.

\section{Statechart Operators}

Our method for statechart synthesis is based on an algebraic framework for statechart composition. This framework is inspired by the algebraic composition of UML2.0 sequence diagrams [44]. We have formalized three statechart operators: $\mathbf{s e q}_{\mathbf{s}}, \mathbf{a l t}_{\mathbf{s}}$ and $\mathbf{l o o p}_{\mathrm{s}}$ respectively for the sequencing, alternation, and the iteration of statecharts. In the rest of this subsection, we briefly describe these operators; the complete formalization can be found in [44]:

- Sequence $\left(\mathbf{s e q}_{\mathbf{s}}\right)$ The sequential composition of two statecharts is a statechart that describes the behavior of the first operand followed by the behavior of the second one. Fig. 11 shows the sequential composition of the ST1 and ST2.

- Alternative $\left(\mathbf{a l t} \mathbf{t}_{\mathbf{s}}\right)$ The statechart resulting from the alternative composition describes a choice between the behaviors of its operands. See for example ST1 alt $\mathbf{t}_{\mathbf{s}}$ ST2 in Fig. 11. 
- Loop $\left(\mathbf{l o o p}_{\mathbf{s}}\right)$ This operator defines iteration of a statechart. Fig. 11 shows the iteration of the ST2.

As for sequence diagrams, we algebraically describe statechart composition with reference expressions.

Definition.6. A Reference expression for statecharts (noted REST hereafter) is an expression of the form:

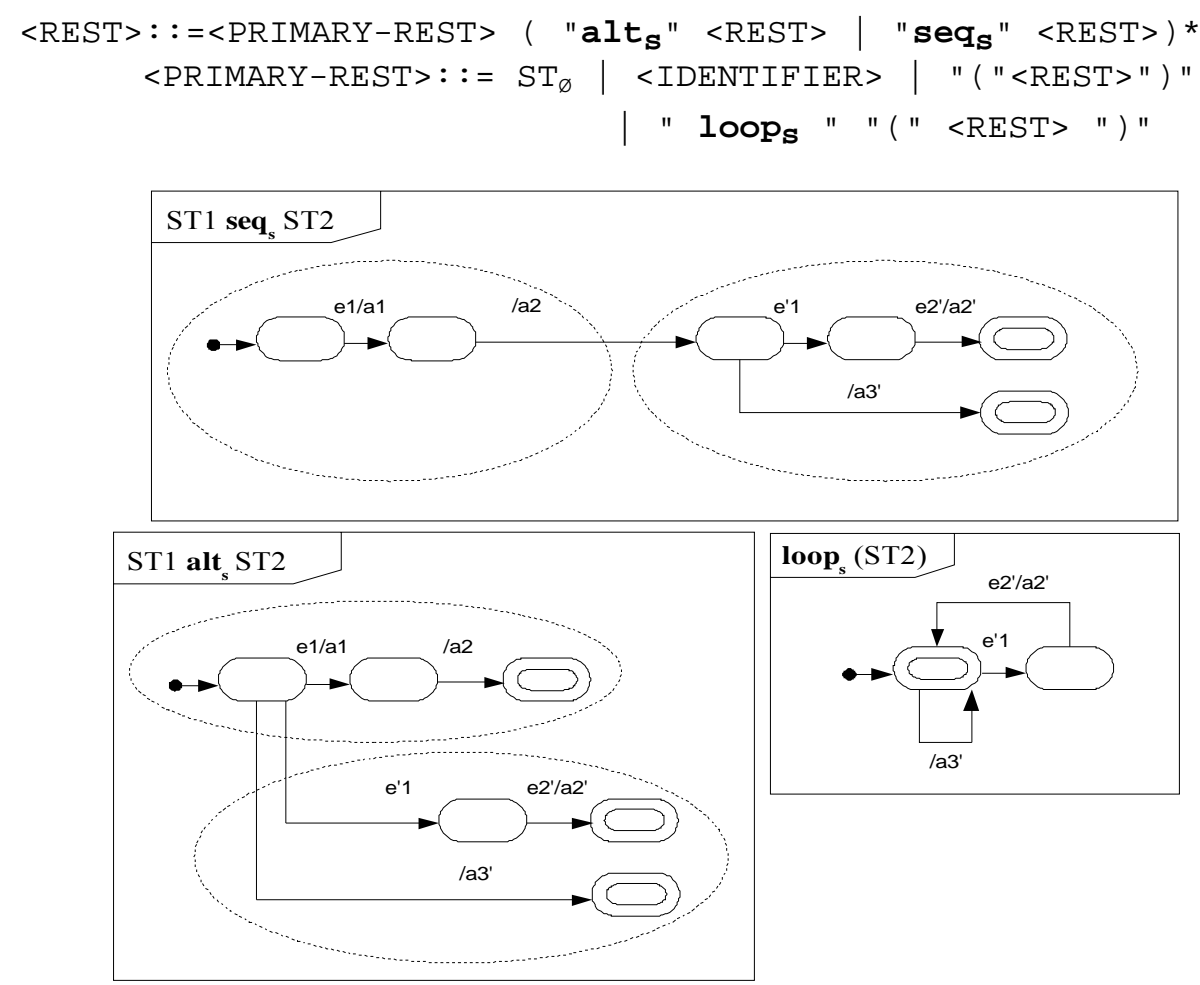

Fig.11. Statechart operators.

\section{Synthesis process}

Using our algebraic framework for statecharts, translating product UML sequence diagrams to statecharts is defined in two steps: synthesis from basic sequence diagrams and synthesis from combined sequence diagrams. The next paragraphs describe these two steps.

Synthesis from basic sequence diagrams. In the first step of our synthesis method we generate statecharts from all basic sequence diagrams in the product line. This step is based on an algorithm generating a statechart $\mathrm{P}(\mathrm{SD}, 0)$ depicting the behavior of each object 0 in each basic SD SD. We do not detail here the algorithm computing $P(S D, 0)$, which 
can be found in [44]. To summarize, this algorithm uses projections of SDs on object lifelines to generate the statecharts. Receptions in the SD become events in the statechart and emissions become actions. For a transition associated with a reception, the action part will be void, and for transitions associated with actions, the event part will be empty. The generated statechart contains a single junction state which corresponds to the state reached when all events situated on an object lifeline have been executed. When an object does not participate in a basic SD, the algorithm generates an empty statechart. Fig. 12 illustrates the synthesis of the statechart associated with the Bank object from the Deposit basic SD.

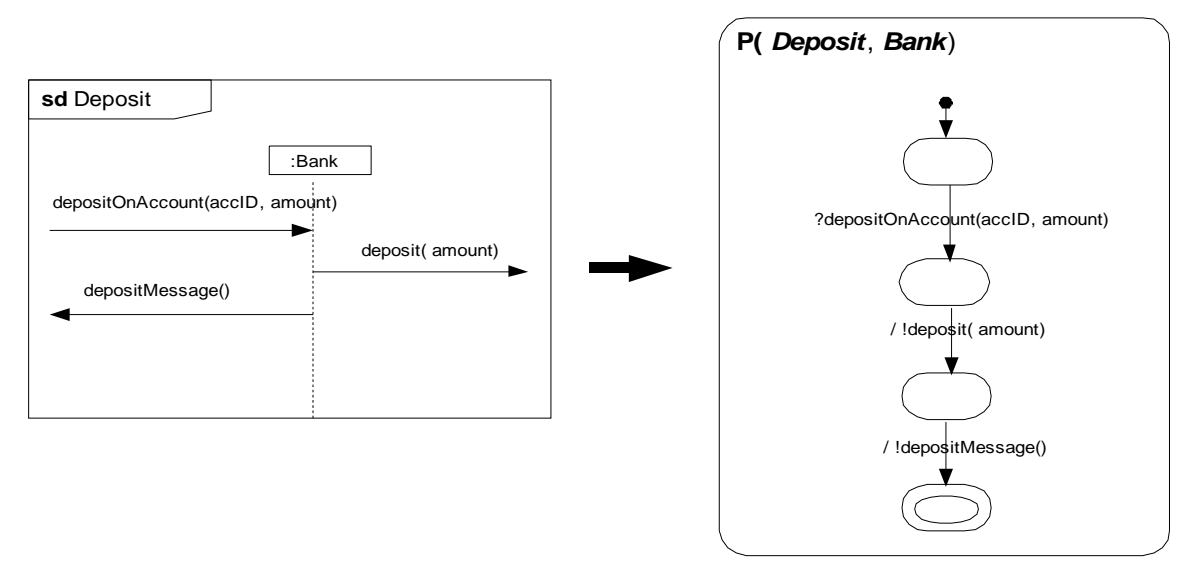

Fig.12. Statechart sythesis from basic SD.

Fig. 13 shows the flat statecharts generated from the twelve basic SDs from Fig. 9 for the Bank object.

Synthesis from combined sequence diagrams. Once we have obtained a collection of statecharts through projections of basic SDs, we now deal with combined SDs. Our method is based on the correspondence between interaction operators and statecharts operators and it allows constructing RESTs from RESDs [44]. For each object 0, a REST is constructed by replacing in the RESD seq, alt, and loop respectively by statecharts operators $\mathbf{s e q}_{\mathrm{s}}, \mathbf{a l t}_{\mathrm{s}}$ and $\mathbf{l o o p}_{\mathrm{s}}$, and each reference to a SD S by the statechart $\mathrm{P}(\mathrm{S}, \mathbf{0})$. From the REST obtained, a statechart can be built using statechart composition operators.

Let us apply this construction method to the combined SD for the BS2 product. The Bank's REST, called REST ${ }_{\mathrm{BS} 2}$ is described below. Fig. 14 shows the statechart obtained from this REST.

REST $_{\text {BS2 }}=$ loop $_{s}(P($ Deposit, Bank $)$ alt $s$ ( $P($ CreateAccount, Bank $)$ 
seqs ( $P($ CreateAccountok, Bank) alts $P($ CreateAccountFailed, Bank )) )

alt $_{\text {s }}$ ( $P$ (WithdrawWithoutLimit, Bank) seq ( (WithdrawOk, Bank)

alts $\mathrm{P}($ WithdrawFailed, Bank))))

The same method can be applied for the BS4 product. Its reference expression $E_{B S 4}$ is transformed into the statechart composition expression $\mathrm{REST}_{\mathrm{BS} 4}$ defined below. Fig. 15 shows the Bank statechart obtained from REST ${ }_{\mathrm{BS} 4}$. Note that as BS2 and BS4 differ in the presence or the absence of an overdrawing limit and exchange operations, the synthesized statecharts differ in the transitions which concern these two functionalities. The differences between the statecharts obtained for product BS2 and BS4 are illustrated in Fig. 15 by grey zones.

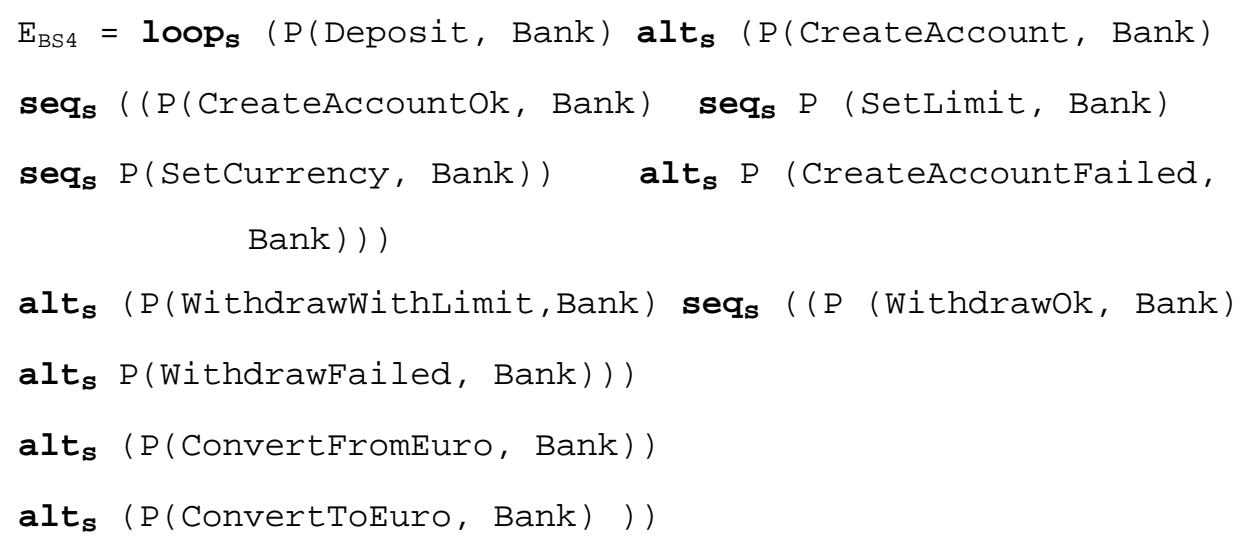




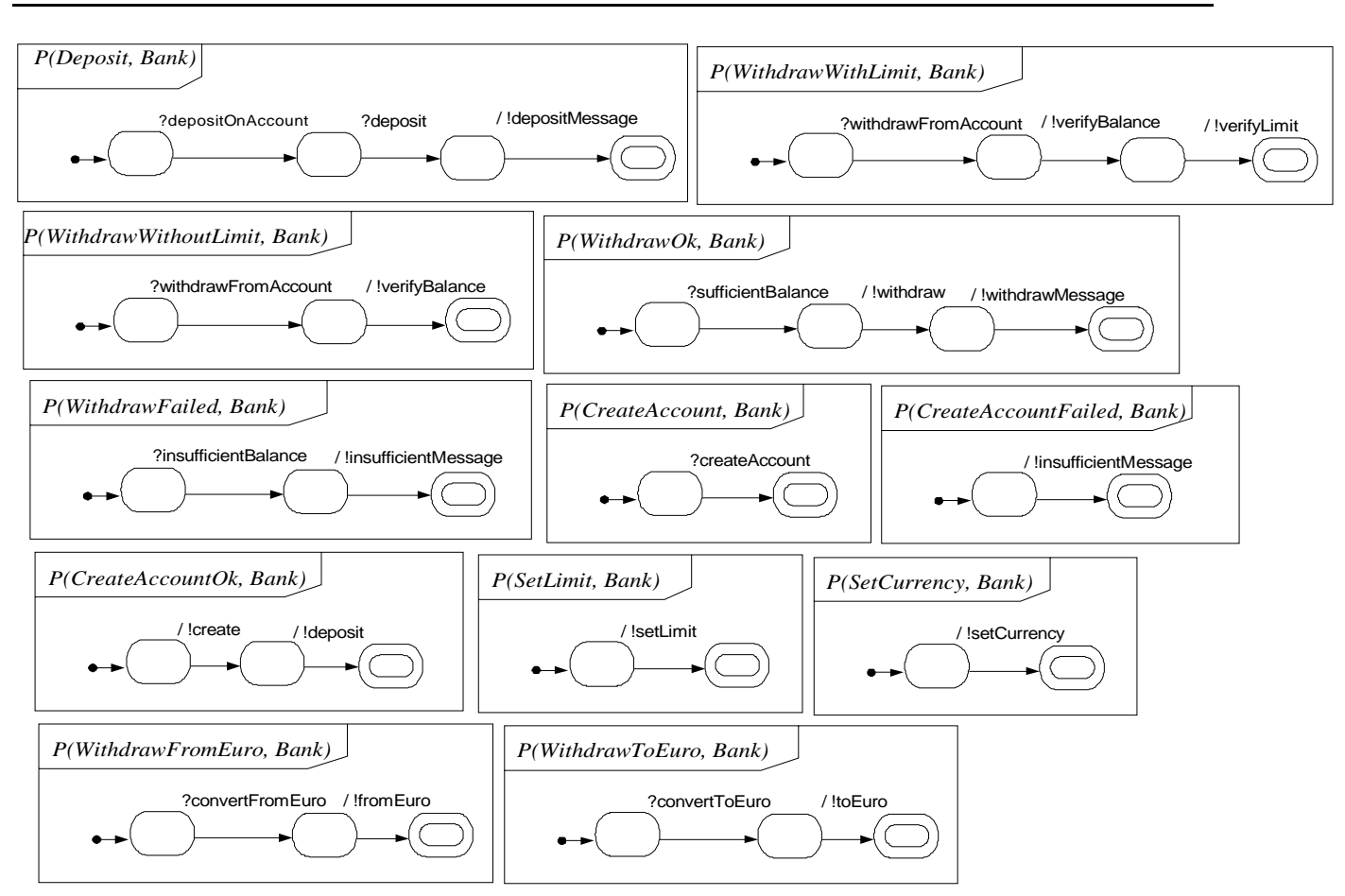

Fig.13. Bank basic statecharts.

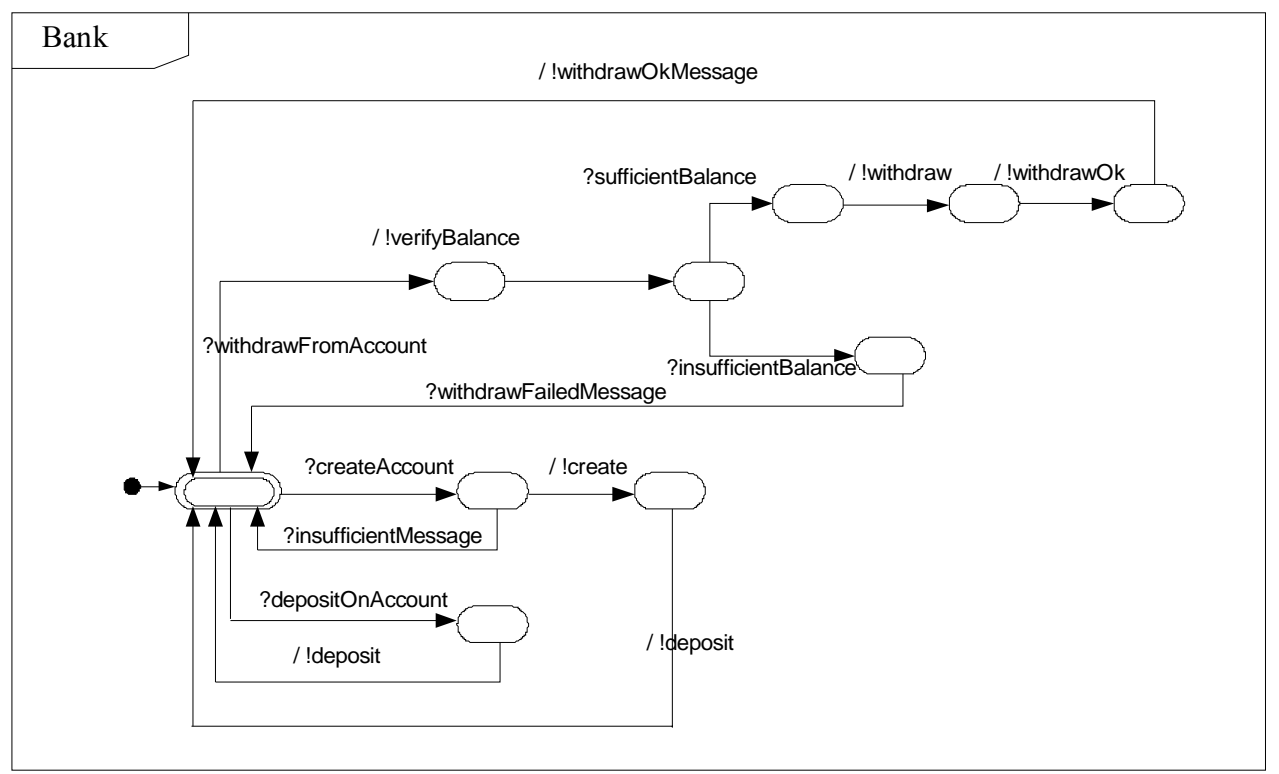

Fig.14. The Bank Statechart in the BS2 product. 


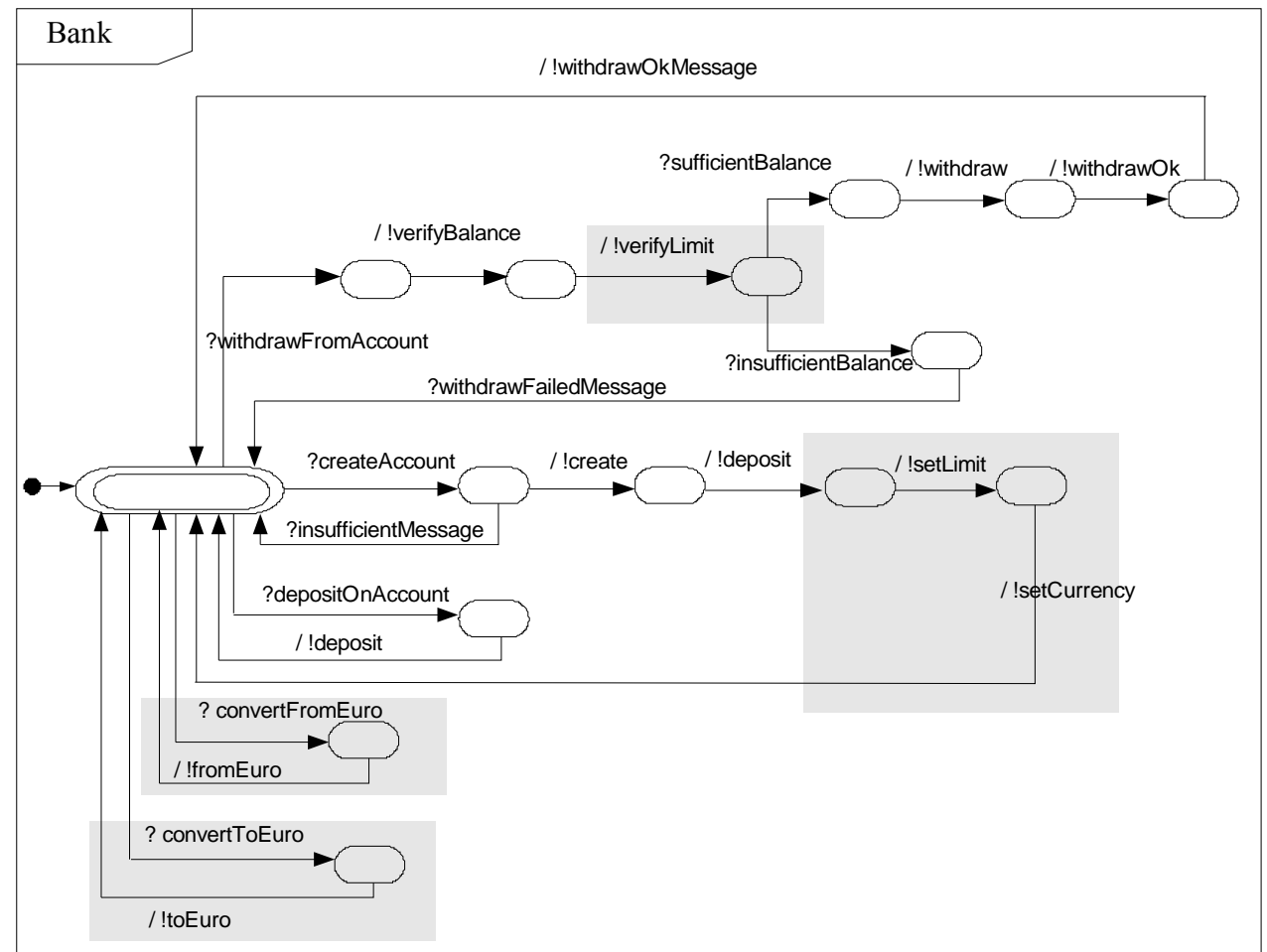

Fig.15. The Bank Statechart in the BS4 product.

\subsubsection{Implementation and validation}

In the context of the ITEA Families [1] project, a prototype tool of the proposed approach has been implemented in Java and is integrated into the Eclipse platform. It is freely available from http://modelware.inria.fr/plibs. UML2.0 sequence diagrams with variability are specified in Eclipse, thanks to the Omondo case tool (see Fig. 16-a) Then RESD-PL are automatically extracted from these diagrams. The prototype implements product expression derivations from RESD-PL according to a given Instance of Decision Models. Then a statechart for a specific object is generated from the derived expression. The generated statecharts can be visualized using the Omondo case tool again (see Fig.16-b). A complete description of the prototype can be found at http://modelware.inria.fr/plibs. 


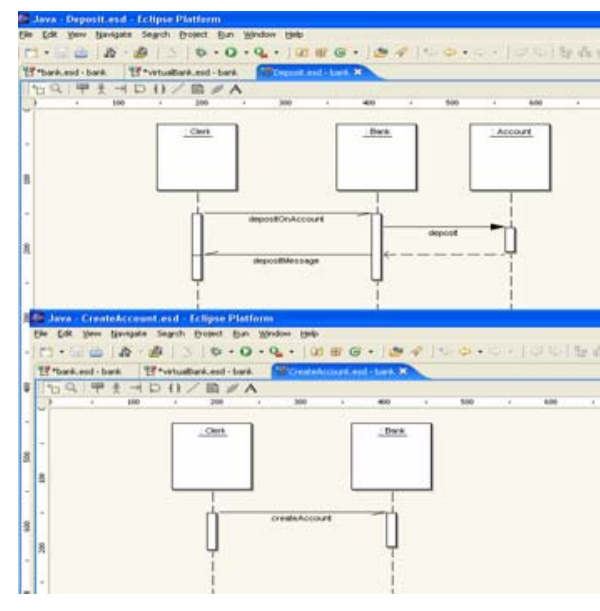

(a) Example of sequence diagrams specification.

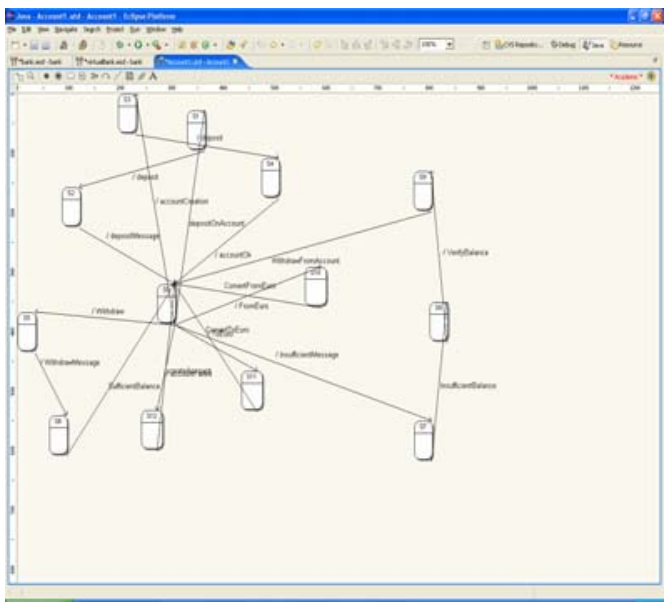

(b) Example of the generated statecharts.

Fig.16. Sequence diagrams and statechart visualization in the PLiBS prototype.

We have used our approach for a complete BPL case study with fourteen basic SDs. Table. 3 shows statistics (number of states and transitions) on the generated statecharts for the Bank object in each BPL member (these statistics show that the generated statechart for the Bank object differs from one product to another). We have also validated our approach on two case studies: The camera Product Line [42] and the auction Product Line [41]. As we noticed in subchapter 999.3, some tools allow generating code from statecharts. We are currently studying code generation from the generated statecharts in our method using existing tools.

Table 3. States and transitions for the generated Bank statechart in the different products.

\begin{tabular}{|l|l|l|}
\hline Product & \# States & \#Transitions \\
\hline BS1 & 12 & 16 \\
BS2 & 10 & 14 \\
BS3 & 13 & 19 \\
BS4 & 15 & 21 \\
\hline
\end{tabular}

\subsection{Related work}

Software Product Line Engineering with the UML has received a lot of attention in recent years. Table 4 summarizes existing work on PL engineering with the UML. Most of these works address variability modeling whereas only two works refer to the product derivation process. 
For variability modeling, many works $[5,17,18,26,37]$ are related to functional models (use cases). Halmans et al. [18] extend use cases with stereotypes to specify variability. Use cases are described using templates. Bertolino et al. [5] introduce tags to describe variability in a textual description of uses cases. In the Chapter "Product Line Use Cases: Scenario-based specification and testing of requirements" of this book, readers can find a detailed description of Bertolino et al.'s work. Maßen et al. [37] extend the UML use case meta-model to support variability. John et al. [26] tailor use-case diagrams and textual use cases to support PL requirements specification. In our work, we do not consider usescases. Even if the textual description through templates, used by the previous works, is a good way to document PL requirements, sequence diagrams are more operational and as shown with our approach detailed design can be generated from them.

There are many works $[3,14,16,10,34,38,27]$ that propose extensions to specify variability in UML static models. However, few works model variability in behavioral models: Gomaa et al. [17] introduce variability in UML collaboration diagrams with three stereotypes <<kernel >>, <<optional >> and <<variant >>. KobrA [3] introduces the stereotype $<<$ variant $>>$ which can be applied to messages in sequence diagrams and to statecharts. The Korba's solution to specify variability in sequence diagrams is difficult to use in practice. Indeed, if all messages in the same sequence diagram are optional, the user should specify all these messages with the stereotype $<<$ variant $>$. This can compromise the readability of the sequence diagram. In contrary, our $<<$ op tionalinteraction $>>$ is applied to the complete sequence diagrams. Flege $[13,14]$ also introduces variability in UML statecharts. Note that all these works only concern UML1.x models.

Concerning product model derivation, only KobrA [3] and Flege [13] refers to this. While we formalized product derivation as UML model transformations, KobrA and Flege do not propose a means to implement derivation. Cerón et al. [8] propose two practices implementing the product architecture derivation. The main assumption in this proposition is: the product line is defined by an engineering assets repository and each product should choose components from this repository to obtain a product-specific architecture.

Haugen et al. [20] also use UML2.0 sequence diagrams to specify behaviors of systems. They introduce a new operator called xatl to distinguish between mandatory and potential behaviors. A potential behavior represents a variant of a mandatory behavior. This is close to our variation construct where interaction variants correspond to the potential behaviors.

In addition to these works, readers can find in the Chapter "Consolidated Product Line Variability Modeling" of this book a complete study about Model Driven Engineering for Software Product Lines. The Chapter also proposes a framework for modeling variability in product lines.

In subchapter 999.3, we have used statechart synthesis from scenarios to derive productspecific behaviors. There are many works on statechart synthesis; however these works only concern single product development (i.e. without consideration for variability). To our knowledge, there are no other works proposing statechart synthesis from software product line scenarios. The next paragraph describes existing works on statechart synthesis in the context of a single product development. There are works that synthesis state- 
charts from UML1.x, from Message Sequence Charts [22] and from Live Sequence Charts [11].

Due to the poor expressive power of UML1.x sequence diagrams, the proposed solutions for statechart synthesis $[29,30,32,40]$ often use additional information or ad-hoc assumptions for managing several scenarios. For example, Whittle et al. [40] enrich messages in sequence diagrams with pre and postconditions given in OCL (Object Constraint Language) which refer to global state variables. State variables identify identical states throughout different scenarios and guide the synthesis process. Our approach does not use variables, and structures the statecharts and transitions based on information provided by lifeline orderings and SD operators. Koskimies et al. [30] use the BiermannKrishnaswamy algorithm [6] which infers programs from traces. This work establishes a correspondence between traces and scenarios and between programs and statecharts. In $[29,32]$ it is also proposed to use interactive algorithms to generate statecharts from UML1.x sequences diagrams.

Several other approaches $[31,35,36]$ study statechart synthesis from Message Sequence charts (MSC) [22], a scenario formalism similar to sequence diagrams. MSCs allow composition of basic scenarios (bMSCs) with High-Level Message Sequence Charts (HMSC). This composition mechanism is very close to that of current SDs in UML2.0 and our approach can be used to generate statecharts from MSCs.

Finally, the Chapter "The ScenTED method for testing Software Product Lines" also uses sequence diagrams but it uses them to derive products-specific test cases from product line requirements and not for statechart synthesis.

Table 4. Existing works on PL engineering with the UML.

\begin{tabular}{|l|c|c|c|c|c|}
\hline \multirow{2}{*}{} & \multicolumn{4}{|c|}{ Variability Modeling } & Product Derivation \\
\cline { 2 - 6 } & $\begin{array}{c}\text { Functional } \\
\text { aspects }\end{array}$ & $\begin{array}{c}\text { Static } \\
\text { aspects }\end{array}$ & $\begin{array}{c}\text { Behavior } \\
\text { aspects }\end{array}$ & $\begin{array}{c}\text { Static } \\
\text { aspects }\end{array}$ & $\begin{array}{c}\text { Behavior } \\
\text { aspects }\end{array}$ \\
\hline Bertolino et al. [5] & $\mathrm{X}$ & & & & \\
\hline Halmans et al.[18] & $\mathrm{X}$ & & & & \\
\hline John et al. [26] & $\mathrm{X}$ & & & & \\
\hline Maßen et al. [37] & $\mathrm{X}$ & & & & \\
\hline Bobak et al. [34] & & $\mathrm{X}$ & $\mathrm{X}$ & & \\
\hline Clauß [9,10] & & $\mathrm{X}$ & & & \\
\hline Gomaa et al. [16, 17] & $\mathrm{X}$ & $\mathrm{X}$ & $\mathrm{X}$ & & \\
\hline Flege [13, 14] & & $\mathrm{X}$ & $\mathrm{X}$ & $\mathrm{X}$ & \\
\hline KobrA [3] & & $\mathrm{X}$ & $\mathrm{X}$ & $\mathrm{X}$ & $\mathrm{X}$ \\
\hline SPLIT-Daisy [27] & & $\mathrm{X}$ & & & \\
\hline Webber [38] & & $\mathrm{X}$ & & & \\
\hline
\end{tabular}




\subsection{Conclusions and Future Research}

In this chapter we have described PL design and derivation techniques building on advanced model transformation technology. Working at the level of UML design models, derivation of both static and behavior aspects were considered. For static aspect derivation, we started from a class diagram modeling the full product line along with a decision model given in the form of a set of concrete factories to build specialized UML models corresponding to the selected products. The challenge of such model manipulation is to be able to transform the model accessing its meta-level and ensuring the integrity of the derived model according to the product line specific constraints.

For behavioral aspects derivation, we started from UML2.0 Sequence Diagrams extended with algebraic constructs to specify variability. We use interpretations of the algebraic expressions to resolve the variability and derive product expressions, which are ultimately transformed into a set of product-specific statecharts. The introduction of variability in behavioral models can be used to factorize common behavioral models in different products, and should then facilitate domain-engineering phases. However, some parts of the synthesis can be reused from one product to another, hence facilitating reuse during application engineering. As discussed in [44], statechart synthesis should be considered more as a step towards implementation rather than as a definitive bridge from user requirements to code.

In the context of the ITEA Families [1] project, prototype tools of the proposed approaches have been implemented. We used MTL (Model Transformation Language) and its related framework UMLAUT-NG for implementing the static aspect derivation. For behavioral aspects, a prototype tool has been implemented in Java and integrated into the Eclipse platform. We used our approach in several case studies; however we hope in the future to use it in an industrial context.

\section{Acknowledgements}

This work has been partially supported by the ITEA project ip02009, FAMILIES in the Eureka $\sum$ ! 2023 Program. We wish to thank Loïc Hélouët for many inspiring discussions. We also gratefully acknowledge the reviews of Stan Bühne, Juan Carlos Dueñas, Timo Käkölä, Kim Lauenroth, Jim Steel, and Patrick Tessier that significantly improved the quality of this chapter.

\section{References}

1. FAMILIES project, http://www.esi.es/Families/, 2003.

2. M. Anastasopoulos and C. Gacek. Implementing Product Line Variabilities. Technical Report IESE Report No. 089.00/E, Version 1.0, IESE, November 2000.

3. C. Atkinson, J. Bayer, C. Bunse, E. Kamsties, O. Laitenberger, R. Laqua, D. Muthig, B. Paech, J. Wüst, and J. Zettel. Component-based Product Line Engineering with UML. Component Software Series. 2001. 
4. L. Bass, P. Clements, and R. Kazman. Software Architecture in Practices. AddisonWesley, first edition, 1998.

5. A. Bertolino, A. Fantechi, S. Gnesi, G. Lami, and A. Maccari. Use Case Description of Requirements for Product Lines. In International Workshop on Requirement Engineering for Product Line (REPL02), pages 12-18, September 2002.

6. A-W. Biermann and R. Krishnaswamy. Constructing programs from example computations. IEEE Transaction on Software Engineering, 2(3):141-153, September 1976.

7. J. Bosch, G. Florijn, D. Greefhorst, J. Kuusela, H. Obbink, and K. Pohl. Variability Issues in Software Product Lines. In Fourth workshop Product Familly Engineering (PFE4), pages 11-19, 2001.

8. R. Cerón, J.L. Arciniegas, J.L. Ruiz, J.C. Dueñas, J. Bermejo, R. Capilla. Architectural modelling in product family context. In Fl'avio Oquendo, Brian Warboys, and Ronald Morrison, editors, EWSA, volume 3047 of Lecture Notes in Computer Science, pages 2542. Springer, 2004.

9. M. Clauß. Generic modeling using UML extensions for variability. In Workshop on Domain Specific Visual Languages at OOPSLA 2001, Tampa Bay, Florida, USA, 2001.

10. M. Clauß. Modeling variability with UML. In GCSE 2001 Young Researchers Workshop, 2001.

11. W. Damm and D. Harel. LSCs: Breathing life into message sequence charts. Formal Methods in System design, 19(1):45-80, 2001.

12. Sybren Deelstra, Marco Sinnema, and Jan Bosch. Product derivation in software product families: a case study. The Journal of Systems and Software, 74(2):173- 194, January 2004.

13. O. Flege. System Family Architecture Description Using the UML. Technical Report IESE-Report No. 092.00/E, IESE, December 2000.

14. O. Flege. Using a decision model to support product line architecture modeling, evaluation, and instantiation. In Proceedings of Product Line Architecture Work-shop. The First Software Product Line Conference (SPLC1), pages 15-20, 2000.

15. E. Gamma, R. Helm, R. Johnson, and J. Vlissides. Design Pattern Elements of Reusable Object-Oriented Software. Addison-Wesley, 1995.

16. H. Gomaa. Object Oriented Analysis and Modeling for Families of Systems with UML. In W.B. Frakes, editor, IEEE International Conference for Software Reuse (ICSR6), pages 89-99, June 2000.

17. H. Gomaa. Modeling Software Product Lines with UML. In Peter Knauber and Giancarlo Succi, editors, International Workshop on Software Product Lines: Economics, Architectures, and Implications (SPLW2), pages 27-31, 2001.

18. G. Halmans and K. Pohl. Communicating the variability of a software-product family to cutomers. Software and System Modeling, 2(1):15-36, 2003.

19. D. Harel. Statecharts: A visual formalism for complex systems. Science of Computer Programming, 8(3):231-274, 1987.

20. O. Haugen and K. Stolen. STAIRS-Steps to Analyze Interactions with Refinement Semantics. In UML Conference UML2003, pages 388-402, October 2003.

21. I-Logix. Rhapsody. http://www.ilogix.com/.

22. ITU-T. Z.120 : Message sequence charts (MSC), November 1999.

23. J-M. Jézéquel. Object Oriented Software Engineering with Eiffel. Addison-Wesley, 1996.

24. J-M. Jézéquel. Object-oriented design of real-time telecom systems. In IEEE International Symposium on Object-oriented Real-time distributed Computing, ISORC'98, Kyoto, Japan, April 1998.

25. J-M. Jézéquel. Reifying configuration management for object-oriented software. In Proceedings of the 20th international conference on Software engineering, pages 240-249. IEEE Computer Society, 1998. 
26. I. John and D. Muthig. Tailoring Use Cases for Product Line Modeling. In International Workshop on Requirement Engineering for Product Line (REPL02), pages 26-32, September 2002

27. W. El Kaim. Managing variability in the LCAT SPLIT/Daisy. In Proceedings of Product Line Architecture Workshop. The First Software Product Line Conference (SPLC1), pages 21-32, 2000.

28. K. Kang, S. Cohen, J. Hess, W. Novak, and S. Peterson. Feature-Oriented Domain Analysis (FODA) Feasibility Study. Technical Report CMU/SEI-90-TR-21, Software Engineering Institute (SEI), November 1990.

29. I. Khriss, M. Elkoutbi, and R. Keller. Automating the synthesis of UML statechart diagrams from multiple collaboration diagrams. In Proceedings of UML'98: Beyond the Notation, pages 115-126, 1998.

30. K. Koskimies, T. Systä, J. Tuomi, and T. Männistö. Automated support for modelling OO software. IEEE Software, 15:87-94, Janu 1998.

31. I. Krüger, R. Grosu, P. Scholz, and M. Broy. From MSCs to statecharts. In Distributed and Parallel Embedded Systems, pages 61-71, 1999.

32. E. Mäkinen and T. Systä. MAS-an interactive synthesizer to support behavioural modeling. In Proceeding of International Conference on Software Engineering (ICSE 2001), 2001.

33. Object Management Group OMG. Unified Modeling Language specification version 2.0: Superstructure. Technical Report pct/03-08-02, OMG, 2003.

34. S. Robak, R. Franczyk, and K. Politowicz. Extending the UML for modeling variability for system families. International Journal of Applied Mathematics Computer Sciences, 12(2):285-298, 2002.

35. S.Uchitel, J.Kramer, and J.Magee. Synthesis of behavioral models from scenarios. IEEE Transaction on Software Engineering, 29(2):99-115, February 2003.

36. S. Uchitel and J. Kramer. A workbench for synthesising behaviour models from scenarios. In Proc. of International Conference on Software Engineering (ICSE 2001), 2001.

37. T. van der Maßen and H. Lichter. Modeling Variability by UML Use Case Diagrams. In International Workshop on Requirement Engineering for Product Line (REPL02), pages 19-25, September 2002.

38. D.L. Webber. The Variation Point Model For Software Product Lines. Ph.D, George Mason University, George Mason University Fairfax, VA, 2001.

39. M.D. Weiss and C.T. Robert Lai. Software Product-Line Engineering: A Family Based Software Development Process. Addison-Wisley, 1999.

40. J.Whittle and J. Schumann. Generating statechart designs from scenarios. In Proceeding of International Conference on Software Engineering (ICSE 2000), 2000.

41. T. Ziadi, Hélouët, and J-M. Jézéquel. Moédélisation de lignes de produits en UML. In Proceedings of LMO 2003, Langages et Modeles a Objets, Vannes, France, Fev. 2003.

42. T. Ziadi, H' Hélouët, and J-M. Jézéquel. Towards a UML profile for software product lines. In Proceedings of the Fifth Internationl Workshop on Product Familly Engineering (PFE-5), volume 3014 of LNCS, pages 129-139. Springer Verlag, 2003.

43. T. Ziadi, L. Hélouët, and J-M. Jézéquel. Modeling behaviors in product lines. In Proceedings of REPL'02 (workshop on Requirements Engineering for Product Lines), Essen, Germany, September 2002.

44. T. Ziadi, L. L. Hélouët, and J-M. Jézéquel. Revisiting statecharts synthesis with an algebraic approach. In International Conference on Software Engineering, ICSE'26, Edinburgh, Scotland, United Kingdom, May 2004.

45. T. Ziadi, J-M. J-M. Jézéquel, and F. Fondement. Product line derivation with UML. In Proceedings Software Variability Management Workshop, Univ. of Groningen Departement of Mathematics and Computing Science, February 2003. 
class diagram, 3

commonality and variability, 1

constraint dependency

constraints, 5

decision model, 7

design

design pattern, 7

feature, 3

model

models transformation, 7 product derivation, 1

scenario, 12

sequence diagram, 12

software product line engineering, 1

UML, 1

OCL, 6

statecharts, 20

UML profile, 3

use case, 30 